\title{
Mathematical modeling of the elastic properties of cubic crystals at small scales based on the Toupin-Mindlin anisotropic first strain gradient elasticity
}

Received: 10 June 2021 / Accepted: 29 August 2021 / Published online: 16 September 2021

(C) The Author(s) 2021

\begin{abstract}
In this work, a mathematical modeling of the elastic properties of cubic crystals with centrosymmetry at small scales by means of the Toupin-Mindlin anisotropic first strain gradient elasticity theory is presented. In this framework, two constitutive tensors are involved, a constitutive tensor of fourth-rank of the elastic constants and a constitutive tensor of sixth-rank of the gradient-elastic constants. First, $3+11$ material parameters $(3$ elastic and 11 gradient-elastic constants), 3 characteristic lengths and $1+6$ isotropy conditions are derived. The 11 gradient-elastic constants are given in terms of the 11 gradient-elastic constants in Voigt notation. Second, the numerical values of the obtained quantities are computed for four representative cubic materials, namely aluminum $(\mathrm{Al})$, copper $(\mathrm{Cu})$, iron $(\mathrm{Fe})$ and tungsten $(\mathrm{W})$ using an interatomic potential (MEAM). The positive definiteness of the strain energy density is examined leading to 3 necessary and sufficient conditions for the elastic constants and 7 ones for the gradient-elastic constants in Voigt notation. Moreover, 5 lattice relations as well as 8 generalized Cauchy relations for the gradient-elastic constants are derived. Furthermore, using the normalized Voigt notation, a tensor equivalent matrix representation of the two constitutive tensors is given. A generalization of the Voigt average toward the sixth-rank constitutive tensor of the gradient-elastic constants is given in order to determine isotropic gradient-elastic constants. In addition, Mindlin's isotropic first strain gradient elasticity theory is also considered offering through comparisons a deeper understanding of the influence of the anisotropy in a crystal as well as the increased complexity of the mathematical modeling.
\end{abstract}

Keywords Strain gradient elasticity · Anisotropy · Higher-rank constitutive tensors - Characteristic lengths . Voigt average

\section{Introduction}

Strain gradient elasticity theories are challenging generalized continuum theories to model crystals at small scales like Ångström-scale, where classical elasticity is not valid and leads to unphysical singularities. The theory of first strain gradient elasticity in its modern form goes back to Toupin [55,56] and Mindlin [41]. Toupin [55] had already given the anisotropic form of strain gradient elasticity, whereas Mindlin [41] derived

Communicated by Andreas Öchsner.

M. Lazar $(\bowtie) \cdot$ E. Agiasofitou · T. Böhlke

Karlsruhe Institute of Technology (KIT), Institute of Engineering Mechanics, 76131 Karlsruhe, Germany

E-mail: markus.lazar@kit.edu

E. Agiasofitou

E-mail: eleni.agiasofitou@kit.edu

T. Böhlke

E-mail: thomas.boehlke@kit.edu 
the corresponding isotropic form. The Mindlin theory of isotropic first strain gradient elasticity [41,43] is often used for the convenience of simplification and the goal of finding analytical solutions. For polycrystals and metamaterials with an isotropic character, Mindlin's isotropic strain gradient elasticity might be a suitable framework. However, for crystals, which are, in general, not isotropic, and for materials possessing a microstructure such an assumption of isotropy is not reliable and can only be considered as an approximation. Therefore, anisotropic strain gradient elasticity is of high relevance for a proper physical modeling of such anisotropic materials. Already, Mindlin [42] pointed out that for cubic crystals with centrosymmetry, anisotropic first strain gradient elasticity should be used. Cubic materials with centrosymmetry cover a big range of important and very useful materials in modern technology and engineering; some of them are aluminum, copper, iron, tungsten, diamond, silicon and germanium. Particularly, silicon and germanium are heavily used as semiconductors in engineering and industrial applications. The understanding of the properties of cubic materials with centrosymmetry and their appropriate modeling with Toupin-Mindlin's anisotropic first strain gradient elasticity is of principal interest and will be investigated in this work.

Anisotropic first strain gradient elasticity theory with general anisotropy includes constitutive tensors of rank four, five and six. Constitutive tensors or matter tensors are tensors representing physical properties of crystals. They have definite orientation within a crystal and must conform to the crystal symmetry [44]. In particular, the higher-rank constitutive tensors are able to model properties like acoustical activity in first strain gradient elasticity and flexoelectricity in first gradient electroelasticity, which cannot be captured by constitutive tensors of lower rank. In this sense, they are physical-property tensors. Field tensors such as the elastic strain tensor and the electric field strength vector do not represent a crystal property and can have any arbitrary orientation in a crystal [44]. Field tensors can be applied and measured in any orientation. The matrix representation of the constitutive tensors of anisotropic first strain gradient elasticity for different elastic symmetries is given by Auffray et al. [4,5]. In the general anisotropic case, a triclinic crystal with symmetry 1 has $210=21+108+171$ material parameters in first strain gradient elasticity. The case of a cubic crystal with centrosymmetry of point group $m \overline{3} m$, which is studied here, has $14=3+11$ material parameters. On the other hand, Mindlin's isotropic first strain gradient elasticity has only $7=2+5$ material parameters. The material parameters of first strain gradient elasticity can be computed from interatomic potentials being a selfconsistent and parameter-free field theory of materials $[1,46]$. The zero-temperature atomistic representation of the fourth-rank, fifth-rank and sixth-rank constitutive tensors in anisotropic first strain gradient elasticity is given by Admal et al. [1]. Shodja et al. [50] have used the Toupin-Mindlin first strain gradient elasticity for cubic crystals of hexoctahedral class to give the analytical expression of the material parameters in terms of the atomic force constants and to evaluate them via ab initio density functional theory (DFT). Moreover, Po et al. [46] have given the Green tensor of Toupin-Mindlin's anisotropic first strain gradient elasticity for centrosymmetric materials.

On the other hand, properties of a material which are intrinsically isotropic can only be modeled by means of tensors of low rank. Let a crystal possess an $N$-fold axis of symmetry $A_{N}$. According to the Hermann theorem [17]: "if a tensor of rank $r$ possesses an axis of symmetry $A_{N}$ with $N>r$, then $A_{N}$ is an axis of isotropy for that tensor". For cubic crystals with centrosymmetry, the minimal rank which is necessary in order to reveal the anisotropy is four, $r=4$, that means tensors of rank four, five and six are anisotropic tensors. Cubic crystals are generally isotropic with respect to second-rank tensorial quantities such as thermal expansion and in crystal optics, cubic crystals are optically isotropic. However, in classical elasticity, a few cubic crystals such as aluminum, tungsten and diamond are isotropic or nearly isotropic and isotropic elasticity can be used as a good approximation (see, e.g., [11]). For example, the reason why tungsten happens to be elastically isotropic despite having cubic lattice symmetry is rooted in its electronic structure and chemical bonding resulting in such values of the elastic constants which satisfy the corresponding isotropy condition of classical elasticity. Then, questions which may arise are: Which are the isotropy conditions in Toupin-Mindlin's anisotropic first strain gradient elasticity for cubic materials with centrosymmetry? Are those conditions fulfilled?

The paper is organized as follows. In Sect. 2, the basic framework of the Toupin-Mindlin anisotropic first strain gradient elasticity with general anisotropy is presented. In Sect. 3, the considered theory is specified to cubic materials with centrosymmetry of point group $m \overline{3} \mathrm{~m}$. Here, the $3+11$ material parameters (3 elastic and 11 gradient-elastic constants), the 3 characteristic lengths and the $1+6$ isotropy conditions are computed. The numerical values of all these quantities are obtained for four representative cubic materials, namely aluminum $(\mathrm{Al})$, copper $(\mathrm{Cu})$, iron $(\mathrm{Fe})$ and tungsten $(\mathrm{W})$. The positive definiteness of the strain energy density is examined. In Sect. 4, Mindlin's isotropic first strain gradient elasticity theory is presented. In Sect. 5, the lattice relations for the gradient-elastic constants as well as the Cauchy relations for the elastic constants and the gradient-elastic constants are derived. In Sect. 6, the normalized Voigt notation is used in order to derive a 
tensor equivalent matrix representation of the two constitutive tensors in first strain gradient elasticity. Based on this representation, the independent eigenvalues of the considered constitutive tensors for the cubic as well as for the isotropic case are derived. In Sect. 7, a generalization of the Voigt average toward the sixthrank constitutive tensor is given in order to determine isotropic gradient-elastic constants. The paper ends with Conclusions which sum up important comparisons between anisotropic and isotropic first strain gradient elasticity for cubic materials with centrosymmetry as well as comparisons between classical elasticity and first strain gradient elasticity. In "Appendix A", the matrix representation in Voigt notation and the conditions of positive definiteness of the two involved constitutive tensors of fourth-rank and sixth-rank using the Sylvester criterion are given.

\section{Anisotropic first strain gradient elasticity with general anisotropy}

Let us consider a three-dimensional infinite elastic body. In the Toupin-Mindlin theory of anisotropic first strain gradient elasticity of form II $[41,42,55,56]$, the strain energy density is given by

$$
\mathcal{W}(\boldsymbol{e}, \nabla \boldsymbol{e})=\frac{1}{2} \mathbb{C}_{i j k l} e_{i j} e_{k l}+\frac{1}{2} \mathbb{D}_{i j m k l n} \partial_{m} e_{i j} \partial_{n} e_{k l}+\mathbb{E}_{i j k l m} e_{i j} \partial_{m} e_{k l},
$$

where $e_{i j}$ denotes the (compatible) elastic strain tensor ${ }^{1}$

$$
e_{i j}=\frac{1}{2}\left(\partial_{i} u_{j}+\partial_{j} u_{i}\right)
$$

which is given in terms of the gradient of the displacement vector $\boldsymbol{u}$. The partial derivative $\partial / \partial x_{k}$ with respect to the spatial coordinate $x_{k}$ is denoted by $\partial_{k}$, and the indices run from 1 to $3, i, j, k, l, m, n=1, \ldots, 3$. Here and in the following all tensor components refer to a Cartesian coordinate system. Thus, in first strain gradient elasticity, the strain energy density (1) is given in terms of the elastic strain tensor $\boldsymbol{e}$ and the gradient of the elastic strain tensor $\nabla \boldsymbol{e}$, which is a hyperstrain tensor sometimes also called double strain tensor (see, e.g., $[27,28]$ ). The (compatible) elastic strain tensor (2) satisfies the compatibility condition [25]

$$
\epsilon_{m k i} \epsilon_{n l j} \partial_{k} \partial_{l} e_{i j}=0
$$

where $\epsilon_{m k i}$ is the Levi-Civita tensor.

In Eq. (1), $\mathbb{C}$ or $\mathbb{C}_{i j k l}$ is the fourth-rank constitutive tensor (matter tensor) of the elastic constants possessing the minor symmetries

$$
\mathbb{C}_{i j k l}=\mathbb{C}_{j i k l}=\mathbb{C}_{i j l k}
$$

and the major symmetry

$$
\mathbb{C}_{i j k l}=\mathbb{C}_{k l i j}
$$

and has 21 independent components. In short notation ${ }^{2}$, the symmetries of the constitutive tensor $\mathbb{C}_{i j k l}$ read

$$
\mathbb{C}_{i j k l} \equiv \mathbb{C}_{(i j) \mid(k l)}
$$

$\mathbb{E}$ or $\mathbb{E}_{i j k l m}$ is the fifth-rank constitutive tensor (matter tensor) of the elastic constants due to the coupling between the elastic strain tensor $e_{i j}$ and the gradient of the elastic strain tensor $\partial_{m} e_{k l}$, possessing only the minor symmetries

$$
\mathbb{E}_{i j k l m}=\mathbb{E}_{j i k l m}=\mathbb{E}_{i j l k m}
$$

\footnotetext{
${ }^{1}$ In the compatible case, the elastic strain tensor coincides with the total strain tensor.

${ }^{2}$ We use the short notation of Schouten [48]. Symmetrization over two indices is denoted by parentheses, $A_{(i j)}:=\left(A_{i j}+\right.$ $\left.A_{j i}\right) / 2$ !, and antisymmetrization by brackets, $B_{[i j]}:=\left(B_{i j}-B_{j i}\right) / 2$ !. The analogous is valid for more indices, as: $C_{(i j k l)}:=$ $\left(C_{i j k l}+C_{j i k l}+C_{i j l k}+21\right.$ more terms $) / 4$ !. Symmetrization over more than 2 indices is, by definition, the normalized sum over all possible permutations of the indices involved. If one or more indices are exempted from symmetrization, then they are enclosed by vertical bars, as: $C_{(i|k l| j)}=\left(C_{i k l j}+C_{j k l i}\right) / 2$ !. One vertical bar denotes the major symmetry in Eq. (5).
} 
and has 108 independent components. In short notation, the symmetries of the constitutive tensor $\mathbb{E}_{i j k l m}$ read

$$
\mathbb{E}_{i j k l m} \equiv \mathbb{E}_{(i j)(k l) m} .
$$

$\mathbb{D}$ or $\mathbb{D}_{i j m k l n}$ is the sixth-rank constitutive tensor (matter tensor) of the gradient-elastic constants possessing the minor symmetries

$$
\mathbb{D}_{i j m k l n}=\mathbb{D}_{j i m k l n}=\mathbb{D}_{i j m l k n}
$$

and the major symmetry

$$
\mathbb{D}_{\text {ijmkln }}=\mathbb{D}_{\text {klnijm }}
$$

and has 171 independent components. In short notation, the symmetries of the constitutive tensor $\mathbb{D}_{i j m k l n}$ read

$$
\mathbb{D}_{i j m k l n} \equiv \mathbb{D}_{(i j) m \mid(k l) n} .
$$

The constitutive tensors can be derived from the strain energy density (1) via

$$
\begin{aligned}
\mathbb{C}_{i j k l} & =\frac{\partial^{2} \mathcal{W}}{\partial e_{i j} \partial e_{k l}}, \\
\mathbb{E}_{i j k l m} & =\frac{\partial^{2} \mathcal{W}}{\partial e_{i j} \partial\left(\partial_{m} e_{k l}\right)}, \\
\mathbb{D}_{i j m k l n} & =\frac{\partial^{2} \mathcal{W}}{\partial\left(\partial_{m} e_{i j}\right) \partial\left(\partial_{n} e_{k l}\right)} .
\end{aligned}
$$

Note that the fifth-rank constitutive tensor $\mathbb{E}_{i j k l m}$ is a pseudo-tensor and changes its sign in an inversion of the coordinate system. $\mathbb{E}_{i j k l m}$ vanishes for materials with central symmetry, whereas is nonzero for noncentrosymmetric materials also called as hemitropic or chiral materials. It is called acoustic gyrotropic tensor and is involved in the phenomenon of acoustical activity in certain non-centrosymmetric crystals $[19,47,55]$.

The quantities conjugate to the elastic strain tensor $e_{i j}$ and the gradient of the elastic strain tensor $\partial_{m} e_{i j}$ are the Cauchy stress tensor $\sigma_{i j}$ (in the Mindlin notation) and the double stress tensor $\tau_{i j m}$, respectively. They are defined as

$$
\begin{aligned}
\sigma_{i j} & =\frac{\partial \mathcal{W}}{\partial e_{i j}}, \\
\tau_{i j m} & =\frac{\partial \mathcal{W}}{\partial\left(\partial_{m} e_{i j}\right)}
\end{aligned}
$$

with $\sigma_{i j}=\sigma_{j i}$ and $\tau_{i j m}=\tau_{j i m}$, and it yields $\sigma_{i j} \equiv \sigma_{(i j)}$ and $\tau_{i j m} \equiv \tau_{(i j) m}$. Using the strain energy density (1), the definitions (15) and (16) lead to the constitutive equations

$$
\begin{aligned}
\sigma_{i j} & =\mathbb{C}_{i j k l} e_{k l}+\mathbb{E}_{i j k l n} \partial_{n} e_{k l}, \\
\tau_{i j m} & =\mathbb{E}_{k l i j m} e_{k l}+\mathbb{D}_{i j m k l n} \partial_{n} e_{k l},
\end{aligned}
$$

which are local and linear. Thus, in general anisotropic first strain gradient elasticity, the Cauchy stress tensor $\sigma=\sigma(\boldsymbol{e}, \nabla \boldsymbol{e})$ and the double stress tensor $\tau=\tau(\boldsymbol{e}, \nabla \boldsymbol{e})$ depend on both the elastic strain and the gradient of the elastic strain. It is clear that the specific expression of the Cauchy stress tensor or also called the force stress tensor as well as of the double stress tensor depends on the form of the strain energy density, which is used. For example, in anisotropic second strain gradient elasticity or also called elasticity of grade three, both Cauchy and double stress tensors depend also on the second gradient of the elastic strain (see, e.g., [3]). Moreover, in first strain gradient elasticity, it should be pointed out that the elastic strain tensor $e_{i j}$ and the gradient of the elastic strain tensor $\partial_{m} e_{i j}$ are the field tensors, whereas the Cauchy stress tensor $\sigma_{i j}$ and the double stress tensor $\tau_{\text {jim }}$ are the response field tensors or excitation field tensors. In this sense, the field tensors cause the excitation field tensors in a crystal. Field tensors and response field tensors have the physical meaning of cause and effect, respectively. The response field tensors are related to the field tensors by means of the (local) constitutive equations, which contain the information about the physical properties of the crystal. Local 
means that the response fields at some point $P$ depend exclusively on the field tensors at the very same point $P$.

The total or effective stress tensor $\widehat{\sigma}_{i j}$ of first strain gradient elasticity is defined by (see also [32])

$$
\widehat{\sigma}_{i j}:=\frac{\partial \mathcal{W}}{\partial e_{i j}}-\partial_{m} \frac{\partial \mathcal{W}}{\partial\left(\partial_{m} e_{i j}\right)}
$$

with $\widehat{\sigma}_{i j}=\widehat{\sigma}_{j i}$ or $\widehat{\sigma}_{i j} \equiv \widehat{\sigma}_{(i j)}$. It is easy to see from Eq. (19) that the symmetric character of the total stress tensor is independent of the specific form of the strain energy density in the framework of first strain gradient elasticity. Using Eqs. (15) and (16), the total or effective stress tensor (19) is given by (see also [29,30])

$$
\widehat{\sigma}_{i j}=\sigma_{i j}-\partial_{m} \tau_{i j m},
$$

which using the constitutive equations (17) and (18) reads

$$
\widehat{\sigma}_{i j}=\mathbb{C}_{i j k l} e_{k l}+\left(\mathbb{E}_{i j k l m}-\mathbb{E}_{k l i j m}\right) \partial_{m} e_{k l}-\mathbb{D}_{i j m k l n} \partial_{m} \partial_{n} e_{k l},
$$

including all 3 constitutive tensors $\mathbb{C}_{i j k l}, \mathbb{E}_{i j k l m}$ and $\mathbb{D}_{i j m k l n}$.

In presence of body forces, the Lagrangian density of first strain gradient elasticity is given by

$$
\mathcal{L}=-\mathcal{W}-\mathcal{V}
$$

where the strain energy density $\mathcal{W}$ is given by Eq. (1) and

$$
\mathcal{V}=-u_{i} f_{i}
$$

is the potential a body force density $f_{i}$.

The Euler-Lagrange equations in first strain gradient elasticity (in statics) are given by (see, e.g., [2])

$$
E_{i}(\mathcal{L})=\frac{\partial \mathcal{L}}{\partial u_{i}}-\partial_{j} \frac{\partial \mathcal{L}}{\partial\left(\partial_{j} u_{i}\right)}+\partial_{m} \partial_{j} \frac{\partial \mathcal{L}}{\partial\left(\partial_{m} \partial_{j} u_{i}\right)}=0 .
$$

Equation (24) provides the force equilibrium condition in first strain gradient elasticity in terms of the Cauchy and double stress tensors

$$
\partial_{j}\left(\sigma_{i j}-\partial_{m} \tau_{i j m}\right)+f_{i}=0
$$

or in terms of the total stress tensor

$$
\partial_{j} \widehat{\sigma}_{i j}+f_{i}=0 .
$$

It should be noted that the specific form of the total or effective stress tensor depends also on the considered theory and it is always the quantity that enters in the divergence in the force equilibrium equation. For instance, the form of the total or effective stress tensor in second gradient electroelasticity can be found in [21].

Using Eqs. (2), (17) and (18), the force equilibrium condition (25) can be cast in the following field equation for the displacement vector

$$
L_{i k}^{\mathrm{M}} u_{k}=-f_{i},
$$

where

$$
L_{i k}^{\mathrm{M}}=\mathbb{C}_{i j k l} \partial_{j} \partial_{l}+\left(\mathbb{E}_{i j k l m}-\mathbb{E}_{k l i j m}\right) \partial_{j} \partial_{l} \partial_{m}-\mathbb{D}_{i j m k l n} \partial_{j} \partial_{l} \partial_{m} \partial_{n}
$$

denotes the differential operator of Mindlin's first strain gradient elasticity called Mindlin operator being a differential operator of fourth order. Equation (27) is an inhomogeneous partial differential equation of fourth order for the displacement $u_{k}$ for a given body force density $f_{i}$.

The Mindlin operator $L_{i k}^{\mathrm{M}}$ can be written as a sum

$$
L_{i k}^{\mathrm{M}}=L_{i k}^{(0)}+L_{i k}^{(1)}-L_{i k}^{(2)}
$$


of the (classical) Navier operator $L_{i k}^{(0)}$, a differential operator of second order,

$$
L_{i k}^{(0)}=\mathbb{C}_{i j k l} \partial_{j} \partial_{l}, \quad \text { with } \quad L_{i k}^{(0)}=L_{k i}^{(0)},
$$

a differential operator of third-order $L_{i k}^{(1)}$ appearing in first strain gradient elasticity due to the coupling between strain and strain gradient tensors

$$
L_{i k}^{(1)}=\left(\mathbb{E}_{i j k l m}-\mathbb{E}_{k l i j m}\right) \partial_{j} \partial_{l} \partial_{m}, \quad \text { with } \quad L_{i k}^{(1)}=-L_{k i}^{(1)},
$$

and a differential operator of fourth-order $L_{i k}^{(2)}$ appearing in first strain gradient elasticity due to the higher-order gradients

$$
L_{i k}^{(2)}=\mathbb{D}_{i j m k l n} \partial_{j} \partial_{l} \partial_{m} \partial_{n}, \quad \text { with } \quad L_{i k}^{(2)}=L_{k i}^{(2)} .
$$

Therefore, the Mindlin operator (29) can be decomposed into symmetric and antisymmetric parts according to

$$
L_{i k}^{\mathrm{M}}=L_{(i k)}^{\mathrm{M}}+L_{[i k]}^{\mathrm{M}},
$$

where its symmetric part reads

$$
L_{(i k)}^{\mathrm{M}}=L_{i k}^{(0)}-L_{i k}^{(2)}
$$

and its antisymmetric part reads

$$
L_{[i k]}^{\mathrm{M}}=L_{i k}^{(1)}
$$

\section{Anisotropic first strain gradient elasticity for cubic crystals with centrosymmetry of point group $m \overline{\mathbf{3}} m$}

In this section, the anisotropic version of the Toupin-Mindlin first strain gradient elasticity is specified to cubic crystals with centrosymmetry of point group $m \overline{3} m$, which are also called cubic hexoctahedral. In the first subsection, the basic framework of the considered theory is given answering among others the question: How many characteristic lengths can be defined in a natural way (that means appearing in the modified Helmholtz operators which are part of the Mindlin operator) in anisotropic first strain gradient elasticity for cubic materials with centrosymmetry? Section 3.2 deals with the derivation of the involved 11 gradient-elastic constants. The numerical values of the material parameters (elastic and gradient-elastic constants) and of the characteristic lengths are computed for four representative cubic materials leading to important observations. Next, the positive definiteness of the strain energy density is examined and the section ends with the important subject of the investigation of the isotropy conditions.

\subsection{Basic framework}

We consider here a cubic crystal with centrosymmetry. Let the Cartesian coordinate axes $x, y$ and $z$ coincide with the cubic crystal directions [100], [010] and [001], respectively. For cubic crystals of point group $m \overline{3} m$ (cubic hexoctahedral), the fourth-rank constitutive tensor $\mathbb{C}_{i j k l}$, possessing the symmetries (4) and (5), is given by (see, e.g., $[11,53])$

$$
\mathbb{C}_{i j k l}=C_{12} \delta_{i j} \delta_{k l}+C_{44}\left(\delta_{i k} \delta_{j l}+\delta_{i l} \delta_{j k}\right)+\left(C_{11}-C_{12}-2 C_{44}\right) \delta_{i j k l},
$$

with

$$
\delta_{i j k l}=\sum_{s=1}^{3} e_{i}^{(s)} e_{j}^{(s)} e_{k}^{(s)} e_{l}^{(s)}
$$


where $\boldsymbol{e}^{(1)}, \boldsymbol{e}^{(2)}, \boldsymbol{e}^{(3)}$ are the (orthogonal) unit vectors of the cubic system. Because the coordinate system coincides with the cubic system, it yields $\delta_{i j k l}=1$ if $i=j=k=l$ and $\delta_{i j k l}=0$ otherwise [11]. In Eq. (36), $C_{11}, C_{12}$ and $C_{44}$ are the 3 independent elastic constants of a cubic crystal (in Voigt notation, see Sect. 3.2) and $\delta_{i j}$ is Kronecker's delta. Following Hirth and Lothe [18], the pre-factor of $\delta_{i j k l}$ in Eq. (36) might be used to define an anisotropy factor for cubic crystals

$$
H=2 C_{44}+C_{12}-C_{11} .
$$

For centrosymmetric crystals, the fifth-rank constitutive tensor $\mathbb{E}_{i j k l m}$ vanishes

$$
\mathbb{E}_{i j k l m}=0 \text {. }
$$

The sixth-rank constitutive tensor $\mathbb{D}_{i j m k l n}$, possessing the symmetries (9) and (10), is given by

$$
\begin{aligned}
\mathbb{D}_{i j m k l n}= & \frac{a_{1}}{2}\left(\delta_{i j} \delta_{k m} \delta_{l n}+\delta_{i j} \delta_{k n} \delta_{l m}+\delta_{k l} \delta_{i m} \delta_{j n}+\delta_{k l} \delta_{i n} \delta_{j m}\right)+2 a_{2} \delta_{i j} \delta_{k l} \delta_{m n} \\
& +\frac{a_{3}}{2}\left(\delta_{j k} \delta_{i m} \delta_{l n}+\delta_{i k} \delta_{j m} \delta_{l n}+\delta_{i l} \delta_{j m} \delta_{k n}+\delta_{j l} \delta_{i m} \delta_{k n}\right)+a_{4}\left(\delta_{i l} \delta_{j k} \delta_{m n}+\delta_{i k} \delta_{j l} \delta_{m n}\right) \\
& +\frac{a_{5}}{2}\left(\delta_{j k} \delta_{i n} \delta_{l m}+\delta_{i k} \delta_{j n} \delta_{l m}+\delta_{j l} \delta_{k m} \delta_{i n}+\delta_{i l} \delta_{k m} \delta_{j n}\right) \\
& +a_{6}\left(\delta_{i k} \delta_{j l m n}+\delta_{i l} \delta_{j k m n}+\delta_{j k} \delta_{i l m n}+\delta_{j l} \delta_{i k m n}\right) \\
& +a_{7}\left(\delta_{k m} \delta_{i j l n}+\delta_{l m} \delta_{i j k n}+\delta_{i n} \delta_{j k l m}+\delta_{j n} \delta_{i k l m}\right) \\
& +a_{8} \delta_{m n} \delta_{i j k l}+a_{9}\left(\delta_{i j} \delta_{k l m n}+\delta_{k l} \delta_{i j m n}\right) \\
& +a_{10}\left(\delta_{i m} \delta_{j k l n}+\delta_{j m} \delta_{i k l n}+\delta_{k n} \delta_{i j l m}+\delta_{l n} \delta_{i j k m}\right) \\
& +a_{11} \delta_{i j k l m n},
\end{aligned}
$$

with

$$
\delta_{i j k l m n}=\sum_{s=1}^{3} e_{i}^{(s)} e_{j}^{(s)} e_{k}^{(s)} e_{l}^{(s)} e_{m}^{(s)} e_{n}^{(s)} .
$$

Here, $a_{1}, \ldots, a_{11}$ are the 11 gradient-elastic constants of a cubic crystal with centrosymmetry and $\delta_{i j k l m n}=1$ if $i=j=k=l=m=n$ and $\delta_{i j k l m n}=0$ otherwise. Note that the sixth-rank constitutive tensor $\mathbb{D}_{i j m k l n}$ given in Eq. (40) consists of an isotropic part with the 5 gradient-elastic constants $a_{1}, \ldots, a_{5}$ and an anisotropic part with the 6 gradient-elastic constants $a_{6}, \ldots, a_{11}$. The isotropic part of Eq. (40) is in agreement with the corresponding one given by Mindlin [41,42]. However, the anisotropic part given by Mindlin [42] does not possess the symmetries (9) and (10), which must be fulfilled. Furthermore, the tensorial structure of the constitutive tensor $\mathbb{D}_{i j m k l n}(40)$ is in accordance with the corresponding one given by Shodja et al. [50] fulfilling the necessary symmetries. However, Shodja et al. [50] use different pre-factors for the isotropic part than the gradient-elastic constants $a_{1}, \ldots, a_{5}$ given in Eq. (40), where we follow Mindlin [41,42] in order to recover in the isotropic limit the constitutive tensor $\mathbb{D}_{i j m k l n}$ of Mindlin's isotropic first strain gradient elasticity [see Eq. (70)].

The strain energy density for cubic materials with centrosymmetry is given by

$$
\mathcal{W}(\boldsymbol{e}, \nabla \boldsymbol{e})=\frac{1}{2} \mathbb{C}_{i j k l} e_{i j} e_{k l}+\frac{1}{2} \mathbb{D}_{i j m k l n} \partial_{m} e_{i j} \partial_{n} e_{k l},
$$

where the constitutive tensors $\mathbb{C}_{i j k l}$ and $\mathbb{D}_{i j m k l n}$ are given by Eqs. (36) and (40), respectively. The corresponding constitutive equations are given by

$$
\begin{aligned}
\sigma_{i j} & =\mathbb{C}_{i j k l} e_{k l}, \\
\tau_{i j m} & =\mathbb{D}_{i j m k l n} \partial_{n} e_{k l} .
\end{aligned}
$$

Using the constitutive tensors for a cubic crystal with centrosymmetry, Eqs. (36) and (40), the Cauchy stress tensor (43) is explicitly written in terms of the 3 elastic constants $C_{11}, C_{12}$ and $C_{44}$ as

$$
\sigma_{i j}=C_{12} \delta_{i j} e_{k k}+2 C_{44} e_{i j}+\left(C_{11}-C_{12}-2 C_{44}\right) \delta_{i j k l} e_{k l}
$$


and the double stress tensor (44) is explicitly written in terms of the 11 gradient-elastic constants as

$$
\begin{aligned}
\tau_{i j m}= & \frac{a_{1}}{2}\left(\delta_{i m} \partial_{j} e_{k k}+\delta_{j m} \partial_{i} e_{k k}+2 \delta_{i j} \partial_{k} e_{m k}\right)+2 a_{2} \delta_{i j} \partial_{m} e_{k k} \\
& +a_{3}\left(\delta_{i m} \partial_{k} e_{j k}+\delta_{j m} \partial_{k} e_{i k}\right)+2 a_{4} \partial_{m} e_{i j}+a_{5}\left(\partial_{i} e_{j m}+\partial_{j} e_{i m}\right) \\
& +2 a_{6}\left(\delta_{j k m n} \partial_{n} e_{i k}+\delta_{i k m n} \partial_{n} e_{j k}\right)+a_{7}\left(2 \delta_{i j k n} \partial_{n} e_{k m}+\delta_{i k l m} \partial_{j} e_{k l}+\delta_{j k l m} \partial_{i} e_{k l}\right) \\
& +a_{8} \delta_{i j k l} \partial_{m} e_{k l}+a_{9}\left(\delta_{i j} \delta_{k l m n} \partial_{n} e_{k l}+\delta_{i j m n} \partial_{n} e_{k k}\right) \\
& +a_{10}\left(\delta_{i m} \delta_{j k l n} \partial_{n} e_{k l}+\delta_{j m} \delta_{i k l n} \partial_{n} e_{k l}+2 \delta_{i j k m} \partial_{l} e_{k l}\right)+a_{11} \delta_{i j k l m n} \partial_{n} e_{k l} .
\end{aligned}
$$

In this way, if we substitute the Cauchy stress tensor (45) and the double stress tensor (46) into Eq. (20), then we obtain the total or effective stress tensor (20) explicitly written in terms of the 3 elastic and 11 gradient-elastic constants as follows

$$
\begin{aligned}
\widehat{\sigma}_{i j}= & C_{12} \delta_{i j} e_{k k}+2 C_{44} e_{i j}+\left(C_{11}-C_{12}-2 C_{44}\right) \delta_{i j k l} e_{k l}-a_{1}\left(\partial_{i} \partial_{j} e_{k k}+\delta_{i j} \partial_{m} \partial_{k} e_{m k}\right) \\
& -2 a_{2} \delta_{i j} \Delta e_{k k}-a_{3}\left(\partial_{i} \partial_{k} e_{j k}+\partial_{j} \partial_{k} e_{i k}\right)-2 a_{4} \Delta e_{i j}-a_{5}\left(\partial_{k} \partial_{i} e_{j k}+\partial_{k} \partial_{j} e_{i k}\right) \\
& -2 a_{6}\left(\delta_{j k m n} \partial_{m} \partial_{n} e_{i k}+\delta_{i k m n} \partial_{m} \partial_{n} e_{j k}\right)-a_{7}\left(2 \delta_{i j k n} \partial_{m} \partial_{n} e_{k m}+\delta_{i k l m} \partial_{m} \partial_{j} e_{k l}+\delta_{j k l m} \partial_{m} \partial_{i} e_{k l}\right) \\
& -a_{8} \delta_{i j k l} \Delta e_{k l}-a_{9}\left(\delta_{i j} \delta_{k l m n} \partial_{m} \partial_{n} e_{k l}+\delta_{i j m n} \partial_{m} \partial_{n} e_{k k}\right) \\
& -a_{10}\left(\delta_{j k l n} \partial_{i} \partial_{n} e_{k l}+\delta_{i k l n} \partial_{j} \partial_{n} e_{k l}+2 \delta_{i j k m} \partial_{m} \partial_{l} e_{k l}\right)-a_{11} \delta_{i j k l m n} \partial_{m} \partial_{n} e_{k l},
\end{aligned}
$$

where $\Delta$ indicates the Laplacian.

Next, we give the necessary differential operators for a cubic crystal with centrosymmetry. In particular, using Eq. (36), the (classical) Navier operator $L_{i k}^{(0)}$, Eq. (30), is explicitly expressed in terms of the 3 elastic constants as follows

$$
L_{i k}^{(0)}=\left(C_{12}+2 C_{44}\right) \partial_{i} \partial_{k}+C_{44}\left(\delta_{i k} \Delta-\partial_{i} \partial_{k}\right)+\left(C_{11}-C_{12}-2 C_{44}\right) \delta_{i j k l} \partial_{j} \partial_{l}
$$

and using Eq. (40), the differential operator $L_{i k}^{(2)}$, Eq. (32), is explicitly written in terms of the 11 gradient-elastic constants as

$$
\begin{aligned}
L_{i k}^{(2)}= & 2\left(a_{1}+a_{2}+a_{3}+a_{4}+a_{5}\right) \partial_{i} \partial_{k} \Delta \\
& +\frac{1}{2}\left(a_{3}+2 a_{4}+a_{5}\right)\left(\delta_{i k} \Delta-\partial_{i} \partial_{k}\right) \Delta \\
& +\left(a_{6}+2 a_{7}+a_{8}+2 a_{10}\right) \delta_{i j k l} \partial_{j} \partial_{l} \Delta \\
& +a_{6} \delta_{i k} \delta_{j l m n} \partial_{j} \partial_{l} \partial_{m} \partial_{n}+a_{11} \delta_{i j k l m n} \partial_{j} \partial_{l} \partial_{m} \partial_{n} \\
& +\left(a_{6}+a_{7}+a_{9}+a_{10}\right)\left(\delta_{k l m n} \partial_{i}+\delta_{i l m n} \partial_{k}\right) \partial_{l} \partial_{m} \partial_{n} .
\end{aligned}
$$

For cubic materials with centrosymmetry, the Mindlin operator $L_{i k}^{\mathrm{M}}$, Eq. (29), is reduced to

$$
L_{i k}^{\mathrm{M}}=L_{i k}^{(0)}-L_{i k}^{(2)}
$$

Using Eqs. (48) and (49), the Mindlin operator for cubic materials with centrosymmetry [Eq. (50)] is explicitly written

$$
\begin{aligned}
L_{i k}^{\mathrm{M}}= & \left(C_{12}+2 C_{44}\right)\left[1-\ell_{1}^{2} \Delta\right] \partial_{i} \partial_{k}+C_{44}\left[1-\ell_{2}^{2} \Delta\right]\left(\delta_{i k} \Delta-\partial_{i} \partial_{k}\right) \\
& +\left(C_{11}-C_{12}-2 C_{44}\right)\left[1-\ell_{3}^{2} \Delta\right] \delta_{i j k l} \partial_{j} \partial_{l} \\
& -a_{6} \delta_{i k} \delta_{j l m n} \partial_{j} \partial_{l} \partial_{m} \partial_{n}-a_{11} \delta_{i j k l m n} \partial_{j} \partial_{l} \partial_{m} \partial_{n} \\
& -\left(a_{6}+a_{7}+a_{9}+a_{10}\right)\left(\delta_{k l m n} \partial_{i}+\delta_{i l m n} \partial_{k}\right) \partial_{l} \partial_{m} \partial_{n} .
\end{aligned}
$$


In Eq. (51), one can see that there appear 3 modified Helmholtz operators $\left[1-\ell_{I}^{2} \Delta\right], I=1,2,3$ with 3 characteristic lengths $\ell_{I}, I=1,2,3$ given by

$$
\begin{aligned}
& \ell_{1}^{2}=\frac{2\left(a_{1}+a_{2}+a_{3}+a_{4}+a_{5}\right)}{C_{12}+2 C_{44}}, \\
& \ell_{2}^{2}=\frac{a_{3}+2 a_{4}+a_{5}}{2 C_{44}}, \\
& \ell_{3}^{2}=\frac{a_{6}+2 a_{7}+a_{8}+2 a_{10}}{C_{11}-C_{12}-2 C_{44}},
\end{aligned}
$$

and 3 non-classical parts (derivatives of fourth order) with no classical counterpart appearing due to the double stress tensor (46). It should be emphasized that the definition of the 3 characteristic lengths comes out from the mathematical structure of the 3 modified Helmholtz operators appearing in the Mindlin operator. In addition, comparing the Mindlin operator for cubic materials with centrosymmetry, Eq. (51), with the Mindlin operator for isotropic materials, Eq. (77), both in first strain gradient elasticity, one can see that only the first two terms of Eq. (51) correspond to the isotropic part and all other terms to the anisotropic part, showing the influence of the anisotropy in the mathematical structure of the equations. It should be mentioned that Shodja et al. [50] defined 6 "characteristic lengths" in a different way.

Let us see some interesting features of the characteristic lengths (52)-(54) for a cubic crystal with centrosymmetry. First of all, note that 9 from the 11 gradient-elastic constants, in particular the $a_{1}, a_{2}, a_{3}, a_{4}$, $a_{5}, a_{6}, a_{7}, a_{8}$ and $a_{10}$, contribute to the characteristic lengths (52)-(54). The first two characteristic lengths $\ell_{1}$ and $\ell_{2}$ satisfying Eqs. (52) and (53) in the isotropic limit lead directly to the characteristic lengths, Eqs. (78) and (79), of Mindlin's isotropic first strain gradient elasticity. The characteristic length that is new and is not appearing in the isotropic case is the length $\ell_{3}$. It is interesting to see that in the characteristic length $\ell_{3}$ the anisotropy factor $H$ naturally appears, so that Eq. (54) can be written as

$$
\ell_{3}^{2}=-\frac{a_{6}+2 a_{7}+a_{8}+2 a_{10}}{H},
$$

showing that $\ell_{3}$ is the characteristic length due to the inherent anisotropy of the cubic crystal. It should be noticed that $\ell_{3}^{2}$, Eq. (55), might be imaginary if $a_{6}+2 a_{7}+a_{8}+2 a_{10}$ and $H$ are of the same sign (positive or negative). Moreover, $H$ remains nonzero for cubic crystals even if the material can be considered as nearly isotropic with respect to the constitutive tensor $\mathbb{C}_{i j k l}$, like for $\mathrm{Al}$ and $\mathrm{W}$ as we will see in Sect. 3.4. In addition, looking at the Mindlin operator (51), we can extract the information that $\ell_{1}$ is the characteristic length for the combination of the elastic constants $C_{12}+2 C_{44}, \ell_{2}$ is the characteristic length for the elastic constant $C_{44}$, and $\ell_{3}$ is the characteristic length for the anisotropy factor $H$. Therefore, there are 3 characteristic lengths for 3 elastic constants $C_{11}, C_{12}$ and $C_{44}$ for cubic crystals with centrosymmetry in anisotropic first strain gradient elasticity, whereas there are 2 characteristic lengths for 2 elastic constants (Lamé constants) in isotropic first strain gradient elasticity [see Eqs. (78) and (79) in Sect. 4].

\subsection{Material parameters}

In this subsection, we express the 11 gradient-elastic constants $a_{1}, a_{2}, \ldots, a_{11}$ in terms of the gradient-elastic constants in Voigt notation $D_{1,1}, D_{1,2}, \ldots, D_{16,17}$. The numerical values of the last ones are computed from interatomic potentials. Next, four representative cubic materials are considered and the numerical values of the 11 gradient-elastic constants $a_{1}, a_{2}, \ldots, a_{11}$ as well as the values of the characteristic lengths $\ell_{1}, \ell_{2}$ and $\ell_{3}$ are calculated.

In what follows, we use the Voigt notation for the constitutive tensor $\mathbb{C}_{i j k l}$, which in its contracted form $C_{\alpha \beta}, \alpha, \beta=1, \ldots, 6$ is a symmetric $6 \times 6$ matrix (see, e.g., [44])

$$
(i j)=11 \rightarrow 1, \quad 22 \rightarrow 2, \quad 33 \rightarrow 3, \quad 23 \rightarrow 4, \quad 13 \rightarrow 5, \quad 12 \rightarrow 6 .
$$

and the Voigt notation for the tensor $\mathbb{D}_{i j m k l n}$, which in its contracted form $D_{\xi, \rho}, \xi, \rho=1, \ldots, 18$ is a symmetric $18 \times 18$ matrix (see, e.g., $[1,4])$ :

$$
\begin{aligned}
(i j m)= & 111 \rightarrow 1, \quad 221 \rightarrow 2, \quad 122 \rightarrow 3, \quad 331 \rightarrow 4, \quad 133 \rightarrow 5, \quad 222 \rightarrow 6, \\
112 & \rightarrow 7, \quad 121 \rightarrow 8, \quad 332 \rightarrow 9, \quad 233 \rightarrow 10, \quad 333 \rightarrow 11, \quad 113 \rightarrow 12, \\
131 & \rightarrow 13, \quad 223 \rightarrow 14, \quad 232 \rightarrow 15, \quad 123 \rightarrow 16, \quad 132 \rightarrow 17, \quad 231 \rightarrow 18 .
\end{aligned}
$$


The 3 independent components of the constitutive tensor $\mathbb{C}_{i j k l}$, Eq. (36), can be written as

$$
\begin{aligned}
& C_{11} \equiv \mathbb{C}_{1111}, \\
& C_{12} \equiv \mathbb{C}_{1122}, \\
& C_{44} \equiv \mathbb{C}_{2323},
\end{aligned}
$$

and the 11 independent components of the constitutive tensor $\mathbb{D}_{i j m k l n}$, Eq. (40), can be expressed in terms of the 11 gradient-elastic constants $a_{1}, a_{2}, \ldots, a_{11}$ :

$$
\begin{aligned}
D_{1,1} & \equiv \mathbb{D}_{11111}=2\left(a_{1}+a_{2}+a_{3}+a_{4}+a_{5}\right)+4\left(a_{6}+a_{7}+a_{10}\right)+a_{8}+2 a_{9}+a_{11}, \\
D_{1,2} & \equiv \mathbb{D}_{111221}=a_{1}+2 a_{2}+a_{9}, \\
D_{1,3} & \equiv \mathbb{D}_{111122}=\frac{a_{1}}{2}+a_{3}+a_{10}, \\
D_{2,2} & \equiv \mathbb{D}_{221221}=2\left(a_{2}+a_{4}\right)+a_{8}, \\
D_{2,3} & \equiv \mathbb{D}_{221122}=\frac{a_{1}}{2}+a_{5}+a_{7}, \\
D_{2,4} & \equiv \mathbb{D}_{221331}=2 a_{2}, \\
D_{2,5} & \equiv \mathbb{D}_{221133}=\frac{a_{1}}{2} \\
D_{3,3} & \equiv \mathbb{D}_{122122}=\frac{a_{3}}{2}+a_{4}+\frac{a_{5}}{2}+a_{6}, \\
D_{3,5} & \equiv \mathbb{D}_{122133}=\frac{a_{3}}{2} \\
D_{16,16} & \equiv \mathbb{D}_{123123}=a_{4}, \\
D_{16,17} & \equiv \mathbb{D}_{123132}=\frac{a_{5}}{2} .
\end{aligned}
$$

It is interesting to observe that the component $D_{1,1}$ [Eq. (59a)] is the only one consisting of all 11 gradientelastic constants $a_{1}, \ldots, a_{11}$. Note that the corresponding relations to Eqs. (59b)-(59k) given in [50], that is, Eqs. (55) in [50] need modification. The Voigt-type matrix representation of the tensors $\mathbb{C}_{i j k l}$ and $\mathbb{D}_{i j m k l n}$ is given in "Appendix A".

Solving the system of Eqs. (59a)-(59k), we obtain the 11 gradient-elastic constants

$$
\begin{aligned}
a_{1} & =2 D_{2,5} \\
a_{2} & =\frac{1}{2} D_{2,4} \\
a_{3} & =2 D_{3,5} \\
a_{4} & =D_{16,16} \\
a_{5} & =2 D_{16,17} \\
a_{6} & =D_{3,3}-D_{3,5}-D_{16,16}-D_{16,17} \\
a_{7} & =D_{2,3}-D_{2,5}-2 D_{16,17} \\
a_{8} & =D_{2,2}-D_{2,4}-2 D_{16,16} \\
a_{9} & =D_{1,2}-D_{2,4}-2 D_{2,5} \\
a_{10} & =D_{1,3}-D_{2,5}-2 D_{3,5} \\
a_{11} & =D_{1,1}-D_{2,2}+2\left(D_{2,4}-D_{1,2}\right)+4\left(D_{16,16}-D_{1,3}-D_{2,3}-D_{3,3}\right)+8\left(D_{2,5}+D_{3,5}+D_{16,17}\right)
\end{aligned}
$$

expressed in terms of the 11 non-vanishing components of the $18 \times 18$ matrix of the gradient-elastic constants in Voigt notation $D_{1,1}, D_{1,2}, \ldots, D_{16,17}$.

In Table 1, the numerical values of the independent elastic and gradient-elastic constants are reported for four representative cubic materials, namely aluminum $(\mathrm{Al})$, copper $(\mathrm{Cu})$, iron $(\mathrm{Fe})$ and tungsten $(\mathrm{W})$, which had been computed in $[1,46]$. These components of the constitutive tensors $\mathbb{C}_{i j k l}$ and $\mathbb{D}_{i j m k l n}$ are given in the Voigt notation. These values are based on the modified-embedded-atom-method (MEAM) interatomic potential by 
Table 1 Elastic and gradient-elastic constants in Voigt notation obtained from the modified-embedded-atom-method (MEAM) interatomic potential by Lee et al. [34] for different cubic crystals

\begin{tabular}{|c|c|c|c|c|}
\hline & $\mathrm{Al}(\mathrm{fcc})$ & $\mathrm{Cu}(\mathrm{fcc})$ & $\mathrm{Fe}(\mathrm{bcc})$ & $\mathrm{W}(\mathrm{bcc})$ \\
\hline$C_{11}\left[\mathrm{eV} / \AA^{3}\right]$ & 0.71366 & 1.09941 & 1.51659 & 3.32405 \\
\hline$C_{12}\left[\mathrm{eV} / \AA^{3}\right]$ & 0.38649 & 0.77973 & 0.86160 & 1.28028 \\
\hline$C_{44}\left[\mathrm{eV} / \AA^{3}\right]$ & 0.19704 & 0.51043 & 0.76096 & 1.01812 \\
\hline$D_{1,1}[\mathrm{eV} / \AA]$ & 1.08551 & 0.65018 & 1.07423 & 3.04998 \\
\hline$D_{1,2}[\mathrm{eV} / \AA]$ & 0.14572 & 0.36659 & 0.32346 & -0.13792 \\
\hline$D_{1,3}[\mathrm{eV} / \AA ⿻$ & 0.15934 & 0.24150 & 0.22850 & 0.49286 \\
\hline$D_{2,2}[\mathrm{eV} / \AA]$ & 0.84221 & 0.73885 & 0.66683 & 1.16373 \\
\hline$D_{2,3}[\mathrm{eV} / \AA]$ & 0.15671 & 0.20651 & 0.03922 & 0.05159 \\
\hline$D_{2,4}[\mathrm{eV} / \AA]$ & 0.71708 & 0.47496 & 0.91961 & 1.75586 \\
\hline$D_{2,5}[\mathrm{eV} / \AA]$ & -0.01143 & -0.04254 & 0.36430 & 0.71878 \\
\hline$D_{3,3}[\mathrm{eV} / \AA]$ & 0.27613 & 0.29055 & 0.50912 & 0.89435 \\
\hline$D_{3,5}[\mathrm{eV} / \AA]$ & -0.12408 & -0.01828 & 0.29905 & 0.09548 \\
\hline$D_{16,16}[\mathrm{eV} / \AA]$ & 0.16786 & 0.03742 & 0.41599 & 0.85853 \\
\hline$D_{16,17}[\mathrm{eV} / \AA]$ & 0.15006 & 0.03739 & 0.38300 & 0.61640 \\
\hline
\end{tabular}

Table 2 Gradient-elastic constants for different cubic crystals

\begin{tabular}{|c|c|c|c|c|}
\hline & $\mathrm{Al}$ (fcc) & $\mathrm{Cu}(\mathrm{fcc})$ & $\mathrm{Fe}(\mathrm{bcc})$ & $\mathrm{W}$ (bcc) \\
\hline$a_{1}[\mathrm{eV} / \AA]$ & -0.02287 & -0.08509 & 0.72859 & 1.43755 \\
\hline$a_{2}[\mathrm{eV} / \AA]$ & 0.35854 & 0.23748 & 0.45980 & 0.87793 \\
\hline$a_{3}[\mathrm{eV} / \AA]$ & -0.24815 & -0.03655 & 0.59810 & 0.19097 \\
\hline$a_{4}[\mathrm{eV} / \AA]$ & 0.16786 & 0.03742 & 0.41599 & 0.85853 \\
\hline$a_{5}[\mathrm{eV} / \AA]$ & 0.30012 & 0.07479 & 0.76600 & 1.23279 \\
\hline$a_{6}[\mathrm{eV} / \AA]$ & 0.08229 & 0.23401 & -0.58892 & -0.67605 \\
\hline$a_{7}[\mathrm{eV} / \AA]$ & -0.13198 & 0.17426 & -1.09107 & -1.89998 \\
\hline$a_{8}[\mathrm{eV} / \AA]$ & -0.21058 & 0.18906 & -1.08476 & -2.30919 \\
\hline$a_{9}[\mathrm{eV} / \AA]$ & -0.54849 & -0.02327 & -1.32474 & -3.33133 \\
\hline$a_{10}[\mathrm{eV} / \AA]$ & 0.41893 & 0.32059 & -0.73389 & -0.41688 \\
\hline$a_{11}[\mathrm{eV} / \AA]$ & -0.19492 & -2.86388 & 8.52704 & 14.79794 \\
\hline
\end{tabular}

Lee et al. [34], which is archived in the OpenKIM repository. Elastic and gradient-elastic constants for this potential were computed using the method described in Admal et al. [1] (see also [46]), and they are available on the KIM repository [35]. The values of the elastic constants $C_{11}, C_{12}$ and $C_{44}$ for $\mathrm{Al}, \mathrm{Cu}$ and $\mathrm{W}$ given in Table 1 are in perfect agreement with the experimental data at $0 \mathrm{~K}$ given in Kittel [23].

Using Eqs. (60a)-(60k) as well as the values of Table 1, we obtain the numerical values of the 11 gradientelastic constants $a_{1}, a_{2}, \ldots, a_{11}$ for the considered cubic crystals, which are given in Table 2 . It is interesting to observe that the gradient-elastic constant $a_{11}$, relevant in the cubic case, gives an important contribution which cannot be neglected. Particularly, for iron and tungsten the value of $a_{11}$ is much greater than the value of the other gradient-elastic constants.

Next, the 3 characteristic lengths, Eqs. (52)-(54), using the values given in Tables 1 and 2, are computed for aluminum, copper, iron and tungsten and reported in Table 3. The following observations can be made:

(i) The characteristic lengths $\ell_{1}$ and $\ell_{2}$ are positive and real for all considered cubic materials.

(ii) The characteristic length (for the anisotropy) $\ell_{3}$ is imaginary for aluminum, copper and tungsten. In this case, if $\ell_{3}$ is substituted, then the modified Helmholtz operator, [1 $\left.-\ell_{3}^{2} \Delta\right]$, becomes a Helmholtz operator (with plus sign). Negative values for the square of the characteristic lengths (due to anisotropy) of cubic materials are also reported in Shodja et al. [50].

(iii) It can be observed that for copper, the characteristic lengths $\ell_{1}$ and $\ell_{2}$ are very small in comparison with the lattice constant $a$, namely $\ell_{1} / a=0.1392$ and $\ell_{2} / a=0.0921$, whereas for aluminum it yields $\ell_{1} / a=0.2946$ and $\ell_{2} / a=0.2449$. The lattice constants in Table 3 have been taken from $[24,51,57,58]$.

(iv) Moreover, if we compare the 3 characteristic lengths, we see that the value of $\ell_{3}$ is much greater than the values of $\ell_{1}$ and $\ell_{2}$ reflecting the influence of the anisotropy. 
Table 3 Characteristic lengths for different cubic crystals

\begin{tabular}{lcccc}
\hline & $\mathrm{Al}(\mathrm{fcc})$ & $\mathrm{Cu}(\mathrm{fcc})$ & $\mathrm{Fe}(\mathrm{bcc})$ & $\mathrm{W}(\mathrm{bcc})$ \\
\hline$\ell_{1}[\AA]$ & 1.19303 & 0.50329 & 1.57824 & 1.66513 \\
$\ell_{2}[\AA]$ & 0.99186 & 0.33281 & 1.20124 & 1.24195 \\
$\ell_{3}[\AA]$ & $2.58079 \mathrm{i}$ & $1.41947 \mathrm{i}$ & 2.47806 & $31.80864 \mathrm{i}$ \\
$a[\AA]$ & 4.04950 & 3.61491 & 2.8665 & 3.1652 \\
\hline
\end{tabular}

\subsection{Positive definiteness of the strain energy density}

The examination of the positive definiteness and semi-definiteness of the strain energy density is important for the establishment of the uniqueness theorem (Kirchhoff [22]) that is the uniqueness of the solution for the displacement field in the field equations (equilibrium equations) as well as for the stability of the material.

A cubic material with centrosymmetry of point group $m \overline{3} m$ is said to be stable if and only if the strain energy density function (42) is nonnegative for all strains and double strains. Thus, the stability condition is expressed by the positive semi-definiteness of the strain energy density

$$
\mathcal{W}(\boldsymbol{e}, \nabla \boldsymbol{e}) \geq 0, \quad \text { for all } \boldsymbol{e} \text { and } \nabla \boldsymbol{e} .
$$

The strain energy density for cubic materials with centrosymmetry (42) is given as a quadratic form with respect to the elastic strain tensor $\boldsymbol{e}$ and the gradient of the elastic strain tensor $\boldsymbol{\nabla} \boldsymbol{e}$ as follows

$$
\begin{aligned}
\mathcal{W} & =\mathcal{W}(\boldsymbol{e})+\mathcal{W}(\nabla \boldsymbol{e}) \\
& =\frac{1}{2} \mathbb{C}_{i j k l} e_{i j} e_{k l}+\frac{1}{2} \mathbb{D}_{i j m k l n} \partial_{m} e_{i j} \partial_{n} e_{k l} \\
& =\frac{1}{2} C_{\alpha \beta} \epsilon_{\alpha} \epsilon_{\beta}+\frac{1}{2} D_{\xi, \rho} \gamma_{\xi} \gamma_{\rho}, \quad \alpha, \beta=1, \ldots, 6, \quad \xi, \rho=1, \ldots, 18,
\end{aligned}
$$

where $C_{\alpha \beta}$ and $D_{\xi, \rho}$ obey the relations (56) and (57), respectively, and $\epsilon_{\alpha}, \alpha=1, \ldots, 6$ and $\gamma_{\xi}, \xi=1, \ldots, 18$ stand for the components of elastic strain tensor $e_{i j}, i, j=1,2,3$ and double strain tensor $\partial_{m} e_{i j}, i, j, m=$ $1,2,3$, respectively (following also the Voigt notation, that is, the relations (56) and (57), respectively). Denoting the two (uncoupled) quadratic parts by

$$
\begin{aligned}
& \mathcal{W}_{\epsilon}=\frac{1}{2} C_{\alpha \beta} \epsilon_{\alpha} \epsilon_{\beta}, \quad \alpha, \beta=1, \ldots, 6, \\
& \mathcal{W}_{\gamma}=\frac{1}{2} D_{\xi, \rho} \gamma_{\xi} \gamma_{\rho}, \quad \xi, \rho=1, \ldots, 18,
\end{aligned}
$$

the condition of positive definiteness of the elastic strain energy density reads

$$
\mathcal{W}=\mathcal{W}_{\epsilon}+\mathcal{W}_{\gamma}>0, \text { for all } \boldsymbol{e} \text { and } \nabla \boldsymbol{e}
$$

Since strains and double strains are uncoupled from each other, we can study $\mathcal{W}_{\epsilon}$ and $\mathcal{W}_{\gamma}$ separately (see, e.g., [14]).

A quadratic form $C_{\alpha \beta} \epsilon_{\alpha} \epsilon_{\beta}$ is called a positive definite form if it is in general positive and can be zero only if all the $\epsilon_{\alpha}$ are zero. There are different but equivalent methods criteria to check the positive definiteness of a quadratic form. A set of necessary and sufficient conditions for a quadratic form to be positive definite is given by the Sylvester theorem; that is, all the leading principal minors of the matrix $C=\left(C_{\alpha \beta}\right)$ are positive. Equivalently, a quadratic form is positive definite if and only if all eigenvalues $\lambda_{\alpha}, \alpha=1, \ldots, 6$ of $\boldsymbol{C}=\left(C_{\alpha \beta}\right)$ are positive: $\lambda_{\alpha}>0, \alpha=1, \ldots, 6$. Analogously, it holds for the quadratic form $D_{\xi, \rho} \gamma_{\xi} \gamma_{\rho}$. In this case, the eigenvalues are $\lambda_{\xi}, \xi=1, \ldots, 18$. It should be mentioned that a quadratic form can be essentially positive without being positive definite [20].

The conditions of positive definiteness impose restrictions to the elastic and gradient-elastic constants. The conditions for the positive definiteness of the matrices $\boldsymbol{C}=\left(C_{\alpha \beta}\right)$ and $\boldsymbol{D}=\left(D_{\xi, \rho}\right)$ are derived in "Appendix A". Consequently, the necessary and sufficient conditions that the strain energy density (42) is positive definite are given by the inequalities (A.3) and (A.7a)-(A.7e), that is, 3 conditions for the elastic constants and 7 conditions for the gradient-elastic constants. The corresponding relations with the nonnegative sign are the 
Table 4 The anisotropy factor $H$ for the fourth-rank constitutive tensor $\mathbb{C}_{i j k l}$ for different cubic materials

\begin{tabular}{lllll}
\hline & $\mathrm{Al}(\mathrm{fcc})$ & $\mathrm{Cu}(\mathrm{fcc})$ & $\mathrm{Fe}(\mathrm{bcc})$ & $\mathrm{W}(\mathrm{bcc})$ \\
\hline$-H=C_{11}-C_{12}-2 C_{44}\left[\mathrm{eV} / \AA^{3}\right]$ & -0.06691 & -0.70118 & -0.86693 & 0.00753 \\
\hline
\end{tabular}

necessary and sufficient conditions in terms of the elastic and gradient-elastic constants for the stability of the material in the Toupin-Mindlin anisotropic first strain gradient elasticity.

In particular, the values of the elastic constants $C_{11}, C_{12}$ and $C_{44}$ given in Table 1 satisfy the conditions (A.3) for a positive definite matrix $C=\left(C_{\alpha \beta}\right)$ for all four considered cubic materials. On the other hand, the 11 gradient-elastic constants in Voigt notation $D_{1,1}, D_{1,2}, \ldots, D_{16,17}$ given in Table 1 satisfy the conditions of positive definiteness (A.7a)-(A.7e) of the matrix $\boldsymbol{D}$ for $\mathrm{Al}$ and $\mathrm{Cu}$ but not for $\mathrm{Fe}$ and $\mathrm{W}^{3}$. It is important to note that many interatomic potentials given in [1] do not lead to a positive definite constitutive tensor $\mathbb{D}_{i j m k l n}$, like for $\mathrm{Fe}$ and W. Nevertheless, the 7 conditions of positive definiteness of the matrix $\boldsymbol{D}$, inequalities (A.7a)(A.7e), might be used to calibrate the interatomic potentials in a way leading to a positive definite matrix D.

\subsection{Isotropy conditions}

In the considered Toupin-Mindlin anisotropic first strain gradient elasticity, the isotropy is related to conditions arising from both constitutive tensors, $\mathbb{C}_{i j k l}$ and $\mathbb{D}_{i j m k l n}$.

The isotropic limit of the constitutive tensor $\mathbb{C}_{i j k l}$, Eq. (36), for a cubic material toward an isotropic material leads to the following isotropy condition for the constitutive tensor $\mathbb{C}_{i j k l}$

$$
C_{11}-C_{12}-2 C_{44}=0,
$$

which is nothing but the isotropy condition for the anisotropy factor, $H=0$, given in Hirth and Lothe [18]. The values of the anisotropy factor $H$ for the considered cubic materials are given in Table 4. From the physical point of view, $H$ is related to the maximum of Young's modulus in the [111] or [100] direction depending on its sign.

The isotropic limit of the constitutive tensor $\mathbb{D}_{i j m k l n}$, Eq. (40), for a cubic material toward an isotropic material leads to the following 6 isotropy conditions for the constitutive tensor $\mathbb{D}_{i j m k l n}$ in terms of the gradientelastic constants

$$
a_{6}=a_{7}=a_{8}=a_{9}=a_{10}=a_{11}=0 .
$$

One could say that the gradient-elastic constants $a_{6}, a_{7}, a_{8}, a_{9}, a_{10}$ and $a_{11}$ are the additional 6 anisotropy factors stemming from the constitutive tensor $\mathbb{D}_{i j m k l n}$. Therefore, the isotropy conditions in anisotropic first strain gradient elasticity are seven, one arising from the fourth-rank constitutive tensor $\mathbb{C}_{i j k l}$ and six arising from the sixth-rank constitutive tensor $\mathbb{D}_{i j m k l n}$.

As it can be seen in Table 2, the gradient-elastic constants $a_{6}, a_{7}, a_{8}, a_{9}, a_{10}$ and $a_{11}$ are far from fulfilling the isotropy conditions (67). Not a single relation of Eq. (67) is fulfilled by the numerical values given in Table 2. Therefore, the following conclusion can be reached: Aluminum and tungsten are nearly isotropic with respect to the fourth-rank constitutive tensor $\mathbb{C}_{i j k l}$, whereas none of the four considered cubic crystals is isotropic with respect to the sixth-rank constitutive tensor $\mathbb{D}_{i j m k l n}$. The obtained result concerning the anisotropic behavior of the sixth-rank constitutive tensor $\mathbb{D}_{i j m k l n}$ is in accordance with the Hermann theorem already discussed in the introduction.

\section{Mindlin's isotropic first strain gradient elasticity}

In this section, we present the basic equations of Mindlin's isotropic first strain gradient elasticity of form II [41] in order for helpful comparisons with the anisotropic version to be done leading to important observations and consequences.

\footnotetext{
${ }^{3}$ For Fe and W, only the 2 inequalities, (A.7c) and (A.7d), are not fulfilled.
} 
4.1 Basic framework

An isotropic constitutive tensor of rank four satisfying the symmetries (4) and (5) reads as

$$
\mathbb{C}_{i j k l}=\lambda \delta_{i j} \delta_{k l}+\mu\left(\delta_{i k} \delta_{j l}+\delta_{i l} \delta_{j k}\right)
$$

where the 2 Lamé constants are given by

$$
\mu=C_{44}=\frac{1}{2}\left(C_{11}-C_{12}\right), \quad \lambda=C_{12}
$$

An isotropic constitutive tensor of rank six satisfying the symmetries (9) and (10) reads as

$$
\begin{aligned}
\mathbb{D}_{i j m k l n}= & \frac{a_{1}}{2}\left(\delta_{i j} \delta_{k m} \delta_{l n}+\delta_{i j} \delta_{k n} \delta_{l m}+\delta_{k l} \delta_{i m} \delta_{j n}+\delta_{k l} \delta_{i n} \delta_{j m}\right)+2 a_{2} \delta_{i j} \delta_{k l} \delta_{m n} \\
& +\frac{a_{3}}{2}\left(\delta_{j k} \delta_{i m} \delta_{l n}+\delta_{i k} \delta_{j m} \delta_{l n}+\delta_{i l} \delta_{j m} \delta_{k n}+\delta_{j l} \delta_{i m} \delta_{k n}\right)+a_{4}\left(\delta_{i l} \delta_{j k} \delta_{m n}+\delta_{i k} \delta_{j l} \delta_{m n}\right) \\
& +\frac{a_{5}}{2}\left(\delta_{j k} \delta_{i n} \delta_{l m}+\delta_{i k} \delta_{j n} \delta_{l m}+\delta_{j l} \delta_{k m} \delta_{i n}+\delta_{i l} \delta_{k m} \delta_{j n}\right),
\end{aligned}
$$

where $a_{1}, a_{2}, a_{3}, a_{4}, a_{5}$ are the 5 gradient-elastic constants in Mindlin's isotropic first strain gradient elasticity theory [41] (see also [31,32,42]).

The strain energy density for isotropic materials in first strain gradient elasticity is given by

$$
\mathcal{W}(\boldsymbol{e}, \nabla \boldsymbol{e})=\frac{1}{2} \mathbb{C}_{i j k l} e_{i j} e_{k l}+\frac{1}{2} \mathbb{D}_{i j m k l n} \partial_{m} e_{i j} \partial_{n} e_{k l},
$$

with the constitutive tensors $\mathbb{C}_{i j k l}$ and $\mathbb{D}_{i j m k l n}$ to be given by Eqs. (68) and (70).

The Cauchy stress tensor $\sigma_{i j}$ reads

$$
\sigma_{i j}=\lambda \delta_{i j} e_{k k}+2 \mu e_{i j}
$$

and the double stress tensor $\tau_{i j m}$ is given by

$$
\begin{aligned}
\tau_{i j m}= & \frac{a_{1}}{2}\left(\delta_{i m} \partial_{j} e_{k k}+\delta_{j m} \partial_{i} e_{k k}+2 \delta_{i j} \partial_{k} e_{m k}\right)+2 a_{2} \delta_{i j} \partial_{m} e_{k k} \\
& +a_{3}\left(\delta_{i m} \partial_{k} e_{j k}+\delta_{j m} \partial_{k} e_{i k}\right)+2 a_{4} \partial_{m} e_{i j}+a_{5}\left(\partial_{i} e_{j m}+\partial_{j} e_{i m}\right) .
\end{aligned}
$$

The total or effective stress tensor $\widehat{\sigma}_{i j}$ reduces to

$$
\begin{aligned}
\widehat{\sigma}_{i j}= & \lambda \delta_{i j} e_{k k}+2 \mu e_{i j}-a_{1}\left(\partial_{i} \partial_{j} e_{k k}+\delta_{i j} \partial_{m} \partial_{k} e_{m k}\right)-2 a_{2} \delta_{i j} \Delta e_{k k} \\
& -a_{3}\left(\partial_{i} \partial_{k} e_{j k}+\partial_{j} \partial_{k} e_{i k}\right)-2 a_{4} \Delta e_{i j}-a_{5}\left(\partial_{k} \partial_{i} e_{j k}+\partial_{k} \partial_{j} e_{i k}\right) .
\end{aligned}
$$

Using Eq. (68), the Navier operator (30) leads to the Navier operator for Mindlin's isotropic first strain gradient elasticity

$$
L_{i k}^{(0)}=(\lambda+2 \mu) \partial_{i} \partial_{k}+\mu\left(\delta_{i k} \Delta-\partial_{i} \partial_{k}\right)
$$

Using Eq. (70), the differential operator $L_{i k}^{(2)}$, Eq. (32), takes in isotropic case the following expression

$$
L_{i k}^{(2)}=2\left(a_{1}+a_{2}+a_{3}+a_{4}+a_{5}\right) \partial_{i} \partial_{k} \Delta+\frac{1}{2}\left(a_{3}+2 a_{4}+a_{5}\right)\left(\delta_{i k} \Delta-\partial_{i} \partial_{k}\right) \Delta
$$


Consequently, from Eq. (29) the isotropic Mindlin operator of first strain gradient elasticity is given by

$$
\begin{aligned}
L_{i k}^{\mathrm{M}} & =L_{i k}^{(0)}-L_{i k}^{(2)} \\
& =(\lambda+2 \mu)\left[1-\ell_{1}^{2} \Delta\right] \partial_{i} \partial_{k}+\mu\left[1-\ell_{2}^{2} \Delta\right]\left(\delta_{i k} \Delta-\partial_{i} \partial_{k}\right),
\end{aligned}
$$

with the 2 characteristic lengths to be given as follows

$$
\begin{aligned}
& \ell_{1}^{2}=\frac{2\left(a_{1}+a_{2}+a_{3}+a_{4}+a_{5}\right)}{\lambda+2 \mu}, \\
& \ell_{2}^{2}=\frac{a_{3}+2 a_{4}+a_{5}}{2 \mu} .
\end{aligned}
$$

Therefore, in Mindlin's isotropic strain gradient elasticity, we have 2 characteristic lengths for 2 elastic constants (Lamé constants) $\lambda$ and $\mu . \ell_{1}$ is the characteristic length for the combination of the Lamé constants $\lambda+2 \mu$, and $\ell_{2}$ is the characteristic length for the Lamé constant $\mu$.

Under isotropy, the isotropy condition for the constitutive tensor $\mathbb{C}_{i j k l}$, Eq. (66), is satisfied, that is

$$
C_{11}-C_{12}-2 C_{44}=0
$$

and the constitutive tensor $\mathbb{D}_{i j m k l n}$ must satisfy the 6 isotropy conditions, Eq. (67), that is,

$$
a_{6}=a_{7}=a_{8}=a_{9}=a_{10}=a_{11}=0 .
$$

In the isotropic case, Eqs. (59a)-(59k) simplify, leading to the following gradient-elastic constants in Voigt notation

$$
\begin{aligned}
D_{1,1} & \equiv \mathbb{D}_{11111}=2\left(a_{1}+a_{2}+a_{3}+a_{4}+a_{5}\right) \\
D_{1,2} & \equiv \mathbb{D}_{111221}=a_{1}+2 a_{2}, \\
D_{1,3} & \equiv \mathbb{D}_{111122}=\frac{a_{1}}{2}+a_{3} \\
D_{2,2} & \equiv \mathbb{D}_{221221}=2\left(a_{2}+a_{4}\right) \\
D_{2,3} & \equiv \mathbb{D}_{221122}=\frac{a_{1}}{2}+a_{5} \\
D_{2,4} & \equiv \mathbb{D}_{221331}=2 a_{2} \\
D_{2,5} & \equiv \mathbb{D}_{221133}=\frac{a_{1}}{2} \\
D_{3,3} & \equiv \mathbb{D}_{122122}=\frac{a_{3}}{2}+a_{4}+\frac{a_{5}}{2} \\
D_{3,5} & \equiv \mathbb{D}_{122133}=\frac{a_{3}}{2} \\
D_{16,16} & \equiv \mathbb{D}_{123123}=a_{4} \\
D_{16,17} & \equiv \mathbb{D}_{123132}=\frac{a_{5}}{2} .
\end{aligned}
$$

Note that Eqs. (82a)-(82k) agree with the corresponding expressions given in Dell'Isola et al. [12] with changed basis vectors. Moreover, Eqs. (82a)-(82k) are in agreement with the corresponding ones given by Ojaghnezhad and Shodja [45]. However, the gradient-elastic constants of the sixth-rank constitutive tensor, $a_{1}, a_{2}$ and $a_{3}$, used in [45] are defined different than in Mindlin [41] which are also used in this work. 
Moreover, Eqs. (60a)-(60k) in the isotropic case reduce to the following gradient-elastic constants

$$
\begin{aligned}
a_{1} & =2 D_{2,5}, \\
a_{2} & =\frac{1}{2} D_{2,4} \\
a_{3} & =2 D_{3,5} \\
a_{4} & =D_{16,16} \\
a_{5} & =2 D_{16,17} \\
0 & =D_{3,3}-D_{3,5}-D_{16,16}-D_{16,17} \\
0 & =D_{2,3}-D_{2,5}-2 D_{16,17} \\
0 & =D_{2,2}-D_{2,4}-2 D_{16,16} \\
0 & =D_{1,2}-D_{2,4}-2 D_{2,5}, \\
0 & =D_{1,3}-D_{2,5}-2 D_{3,5}, \\
0 & =D_{1,1}-D_{2,2}+2\left(D_{2,4}-D_{1,2}\right)+4\left(D_{16,16}-D_{1,3}-D_{2,3}-D_{3,3}\right)+8\left(D_{2,5}+D_{3,5}+D_{16,17}\right) .
\end{aligned}
$$

Thus, in the isotropic approach, first strain gradient elasticity possesses only 5 independent gradient-elastic constants.

Using Eqs. (83f)-(83k), the isotropy conditions (81) can be expressed in terms of the gradient-elastic constants in Voigt notation as follows

$$
\begin{aligned}
& D_{3,3}=D_{3,5}+D_{16,16}+D_{16,17} \\
& D_{2,3}=D_{2,5}+2 D_{16,17} \\
& D_{2,2}=D_{2,4}+2 D_{16,16} \\
& D_{1,2}=D_{2,4}+2 D_{2,5} \\
& D_{1,3}=D_{2,5}+2 D_{3,5} \\
& D_{1,1}=D_{2,4}+2 D_{16,16}+4\left(D_{2,5}+D_{3,5}+D_{16,17}\right),
\end{aligned}
$$

providing additional relations (constraints) between them.

\section{Lattice-theoretical representation of the constitutive tensors in first strain gradient elasticity}

In this section, we derive lattice relations for the constitutive tensor $\mathbb{D}_{i j m k l n}$ and Cauchy relations for the constitutive tensors $\mathbb{C}_{i j k l}$ and $\mathbb{D}_{i j m k l n}$ based on a lattice-theoretical representation. These relations provide important information for the elastic as well as the gradient-elastic constants.

\subsection{Lattice relations}

In a lattice-theoretical approach of the constitutive tensors in first strain gradient elasticity with general anisotropy, the tensors of elastic constants and gradient-elastic constants are represented by (see, e.g., [50,52])

$$
\begin{aligned}
\widehat{\mathbb{C}}_{i j k l} & =-\frac{1}{2 v_{0}} \sum_{R} \Phi_{i j}(\boldsymbol{R}) X_{k} X_{l}, \\
\widehat{\mathbb{E}}_{i j k l m} & =-\frac{1}{4 v_{0}} \sum_{R} \Phi_{i j}(\boldsymbol{R}) X_{k} X_{l} X_{m}, \\
\widehat{\mathbb{D}}_{i j k l m n} & =-\frac{1}{8 v_{0}} \sum_{R} \Phi_{i j}(\boldsymbol{R}) X_{k} X_{l} X_{m} X_{n},
\end{aligned}
$$

where $\Phi_{i j}(\boldsymbol{R})$ are the atomic force constant tensors of second-rank, $v_{0}$ is the volume of the unit cell, and $X_{k}$ is the atomic distance vector. The atomic force constant tensors are equivalent to the components of the Hessian 
matrix which can be obtained by first principles density functional theory (DFT) (see, e.g., [50]). The tensor $\widehat{\mathbb{C}}_{i j k l}$ is the so-called Born-Huang tensor possessing the symmetries

$$
\widehat{\mathbb{C}}_{i j k l}=\widehat{\mathbb{C}}_{(i j) \mid(k l)}
$$

and having 21 independent components. It is well known that the tensor $\widehat{\mathbb{C}}_{i j k l}$ has the same symmetries as the tensor $\mathbb{C}_{i j k l}$ (see [37,38]).

Note that the tensor $\widehat{\mathbb{E}}_{i j k l m}$ possesses the following symmetries

$$
\widehat{\mathbb{E}}_{i j k l m}=\widehat{\mathbb{E}}_{(i j)(k l m)}
$$

having 60 independent components, and the tensor $\widehat{\mathbb{D}}_{i j k l m n}$ possesses the following symmetries

$$
\widehat{\mathbb{D}}_{i j k l m n}=\widehat{\mathbb{D}}_{(i j)(k l m n)}
$$

having 90 independent components.

Moreover, the Born-Huang tensor $\widehat{\mathbb{C}}_{i j k l}$ can be expressed in terms of the tensor of elastic constants $\mathbb{C}_{i k j l}$ as follows

$$
\widehat{\mathbb{C}}_{i j k l}=\frac{1}{2}\left(\mathbb{C}_{i k j l}+\mathbb{C}_{i l j k}\right) .
$$

For the tensor of elastic-gradient constants $\mathbb{D}_{i j m k l n}$, the generalized Born-Huang tensor $\widehat{\mathbb{D}}_{i j k l m n}$ following Shodja et al. [50] reads as

$$
\widehat{\mathbb{D}}_{i j k l m n}=\frac{1}{6}\left(\mathbb{D}_{\text {mniklj }}+\mathbb{D}_{\text {knimlj }}+\mathbb{D}_{\text {klimnj }}+\mathbb{D}_{\text {mlinkj }}+\mathbb{D}_{\text {lnimkj }}+\mathbb{D}_{\text {mkinlj }}\right),
$$

or shortly

$$
\widehat{\mathbb{D}}_{(i j)(k l m n)}=\mathbb{D}_{(m n|i| k l) j} .
$$

The generalized Born-Huang tensor $\widehat{\mathbb{D}}_{i j k l m n}$ is related to only 90 components of the tensor $\mathbb{D}_{i j m k l n}$, and this part is symmetric in the first, second, fourth and fifth indices, $\mathbb{D}_{(m n|i| k l) j}$. The tensor $\widehat{\mathbb{D}}_{i j k l m n}$ has more symmetries than the tensor $\mathbb{D}_{i j m k l n}$. For that reason, only 90 components of the constitutive tensor $\mathbb{D}_{i j m k l n}$ can be computed from a lattice-theoretical approach.

Therefore, we consider that the tensor $\mathbb{D}_{i j m k l n}$ is symmetric in $i, j, k, l$, and then, it has 90 independent components in the case of general anisotropy: $\mathbb{D}_{i j m k l n}=\mathbb{D}_{(i j|m| k l) n}$. Due to the major symmetry (10), the tensor $\mathbb{D}(i j|m| k l) n$ is also symmetric in the indices $m$ and $n$. Then, in the case of cubic materials with centrosymmetry, the constitutive tensor $\mathbb{D}_{i j m k l n}$, Eq. (40), leads to the following 5 relations between the gradient-elastic constants

$$
\begin{aligned}
& a_{1}=a_{3}=a_{5}, \\
& 2 a_{2}=a_{4}, \\
& a_{6}=a_{9}, \\
& a_{7}=a_{10} .
\end{aligned}
$$

Equations (94)-(97) are the 5 lattice relations for the gradient-elastic constants which must be fulfilled in a lattice-theoretical approach of first strain gradient elasticity for cubic materials with centrosymmetry. It can be seen that there are only 6 independent gradient-elastic constants instead of 11 ones for a cubic crystal with centrosymmetry. The lattice relations (94)-(97) are in agreement with the corresponding ones given by Shodja et al. [50]. Moreover, it is interesting to note that the so-called sixth-rank tensor of "dynamic elastic constants" used in lattice dynamics possesses also the symmetries (93) and consequently it has 6 "dynamic elastic constants" for cubic crystals which can be determined from lattice dynamics (see $[13,19,39]$ ).

In the isotropic limit, the corresponding lattice relations for the gradient-elastic constants are Eqs. (94) and (95) leading to 2 independent gradient-elastic constants instead of 5 ones, which is in agreement with the results obtained in $[13,49]$. 


\subsection{Cauchy relations}

Here, we investigate the Cauchy relations for the constitutive tensors $\mathbb{C}_{i j k l}$ and $\mathbb{D}_{i j m k l n}$ in first strain gradient elasticity.

A lattice-theoretical approach for the elastic constants shows (see, e.g., $[37,38])$ that the Cauchy relations are valid when it is provided:

(i) ideal lattice, no defects,

(ii) the interaction forces between the atoms or molecules of a crystal are central forces, as in halite,

(iii) each atom or molecule is a center of symmetry,

(iv) the interaction forces between the building blocks of a crystal can be well approximated by a harmonic potential.

In most elastic bodies, the Cauchy relations for the constitutive tensor $\mathbb{C}_{i j k l}$ are not satisfied (see, e.g., [16]). Nevertheless, halite $(\mathrm{NaCl})$ fulfills nearly the Cauchy relation for $\mathbb{C}_{i j k l}$ (see, e.g., $[11,16]$ ). A study of the violations of the Cauchy relations yields important information about the interatomic forces of elastic bodies. The same assumptions (i)-(iv) should also hold for the "generalized Cauchy relations" for the constitutive tensor $\mathbb{D}_{i j m k l n}$. For central forces $F(\boldsymbol{R})$, the force constant tensors read as (e.g., $\left.[37,38]\right)$

$$
\Phi_{i j}(\boldsymbol{R})=-F(\boldsymbol{R}) X_{i} X_{j} .
$$

- Substituting Eq. (98) into Eq. (85), the Born-Huang tensor for central forces reads

$$
\widehat{\mathbb{C}}_{(i j k l)}=\frac{1}{2 v_{0}} \sum_{R} F(\boldsymbol{R}) X_{i} X_{j} X_{k} X_{l},
$$

being a totally symmetric tensor of rank four, and consequently, it holds

$$
\widehat{\mathbb{C}}_{(i j k l)}=\mathbb{C}_{(i j k l)} \text {. }
$$

$\mathbb{C}_{(i j k l)}$ is a totally symmetric tensor with 15 independent components

$$
\mathbb{C}_{i j k l}=\mathbb{C}_{(i j k l)}
$$

leading to the well-known Cauchy relations for the fourth-rank constitutive tensor $\mathbb{C}_{i j k l}$.

For a cubic crystal with centrosymmetry [see Eq. (36)], Eq. (101) reduces to the Cauchy relation for the elastic constants:

$$
C_{12}=C_{44} \text {. }
$$

Therefore, the number of the independent elastic constants reduces to 2 constants instead of 3 ones.

- Substituting Eq. (98) into Eq. (87), the generalized Born-Huang tensor for central forces reads

$$
\widehat{\mathbb{D}}_{(i j k l m n)}=\frac{1}{8 v_{0}} \sum_{R} F(\boldsymbol{R}) X_{i} X_{j} X_{k} X_{l} X_{m} X_{n},
$$

which is a totally symmetric tensor of rank six, and consequently, it holds

$$
\widehat{\mathbb{D}}_{(i j k l m n)}=\mathbb{D}_{(i j k l m n)} \text {. }
$$

$\mathbb{D}_{(i j m k l n)}$ is a totally symmetric tensor with 28 independent components

$$
\mathbb{D}_{i j m k l n}=\mathbb{D}_{(i j m k l n)}
$$

leading to the generalized Cauchy relations for the sixth-rank constitutive tensor $\mathbb{D}_{i j m k l n}$ in first strain gradient elasticity.

For a cubic crystal with centrosymmetry (see Eq. (40)), Eq. (105) provides the following 8 generalized Cauchy relations for the gradient-elastic constants:

$$
\begin{aligned}
& \frac{a_{1}}{2}=\frac{a_{3}}{2}=\frac{a_{5}}{2}=2 a_{2}=a_{4}, \\
& a_{6}=a_{7}=a_{8}=a_{9}=a_{10} .
\end{aligned}
$$

Through the generalized Cauchy relations, Eqs. (106) and (107), the number of the independent gradientelastic constants reduces to only 3 constants of a lattice instead of 11 ones for a cubic crystal.

In the isotropic limit, Eq. (106) holds and leads to only 1 independent gradient-elastic constant of a lattice instead of 5 ones for an isotropic material. 


\section{Tensor equivalent matrix representation of the constitutive tensors in first strain gradient elasticity}

In this section, the normalized Voigt notation, proposed by Mehrabadi and Cowin [40], Cowin and Mehrabadi [9] in anisotropic elasticity, is used in order to derive a tensor equivalent matrix representation of the constitutive tensors in first strain gradient elasticity (see also [4]).

In the normalized Voigt notation, the stress and the elastic strain, being second-rank tensors in the threedimensional space, are represented as first-rank tensors (vectors) in a six-dimensional space using an orthonormal basis. Analogously, the elasticity tensor, which is a fourth-rank tensor in the three-dimensional space, is represented by a second-rank tensor in a six-dimensional space. Therefore, invariants of the elasticity tensor can be directly computed from the aforementioned matrix representation applying standard matrix formula. In a similar way, third-rank tensors in the three-dimensional space can be represented as first-rank tensors (vectors) in an 18-dimensional space and sixth-rank tensors in three-dimensional space as second-rank tensors in an 18-dimensional space. It should be noted that in the (non-normalized) Voigt notation, the basis is not orthonormal. As a result, the matrix representation of the constitutive tensors cannot be directly used to solve the eigenvalue problem in linear elasticity, important, for example, to determine wave speeds and independent strain modes (see, e.g., $[9,40])$.

\subsection{Normalized Voigt notation: $\tilde{C}_{\alpha \beta}$ and $\tilde{D}_{\xi, \rho}$}

For the calculation of the invariants of a fourth-rank tensor, it is useful to introduce a matrix representation. First, an orthonormal basis $\left\{\boldsymbol{B}_{\alpha}\right\}(\alpha=1, \ldots, 6)$ is introduced for symmetric second-rank tensors. A possible basis is given by

$$
\begin{array}{ll}
\boldsymbol{B}_{1}=\boldsymbol{e}_{1} \otimes \boldsymbol{e}_{1}, & \boldsymbol{B}_{4}=\frac{1}{\sqrt{2}}\left(\boldsymbol{e}_{2} \otimes \boldsymbol{e}_{3}+\boldsymbol{e}_{3} \otimes \boldsymbol{e}_{2}\right), \\
\boldsymbol{B}_{2}=\boldsymbol{e}_{2} \otimes \boldsymbol{e}_{2}, & \boldsymbol{B}_{5}=\frac{1}{\sqrt{2}}\left(\boldsymbol{e}_{1} \otimes \boldsymbol{e}_{3}+\boldsymbol{e}_{3} \otimes \boldsymbol{e}_{1}\right), \\
\boldsymbol{B}_{3}=\boldsymbol{e}_{3} \otimes \boldsymbol{e}_{3}, & \boldsymbol{B}_{6}=\frac{1}{\sqrt{2}}\left(\boldsymbol{e}_{1} \otimes \boldsymbol{e}_{2}+\boldsymbol{e}_{2} \otimes \boldsymbol{e}_{1}\right),
\end{array}
$$

which satisfies

$$
\boldsymbol{B}_{\alpha} \cdot \boldsymbol{B}_{\beta}=\delta_{\alpha \beta}, \quad \alpha, \beta=1, \ldots, 6
$$

with Kronecker symbol $\delta_{\alpha \beta}$ and $\left\{\boldsymbol{e}_{i}\right\}(i=1, \ldots, 3)$ an orthonormal basis in $\mathbb{R}^{3}$. It should be noted that the basis introduced here is not the same as the basis of classical Voigt notation. Using this basis system, secondrank tensors in three-dimensional space are represented as first-rank tensors (vectors) in a six-dimensional space. Analogously, fourth-rank tensors in three-dimensional space are represented as second-rank tensors in a six-dimensional space.

The components of the stress, strain and elasticity tensors are determined by projection onto the basis tensors

$$
\tilde{\sigma}_{\alpha}=\boldsymbol{\sigma} \cdot \boldsymbol{B}_{\alpha}, \quad \tilde{\varepsilon}_{\alpha}=\boldsymbol{e} \cdot \boldsymbol{B}_{\alpha}, \quad \tilde{\boldsymbol{C}}_{\alpha \beta}=\boldsymbol{B}_{\alpha} \cdot \mathbb{C}\left[\boldsymbol{B}_{\beta}\right] .
$$

The classical Hooke's law can then be equivalently expressed by

$$
\boldsymbol{\sigma}=\sum_{\alpha=1}^{6} \tilde{\sigma}_{\alpha} \boldsymbol{B}_{\alpha}=\sum_{\alpha, \beta=1}^{6} \tilde{C}_{\alpha \beta} \tilde{\varepsilon}_{\beta} \boldsymbol{B}_{\alpha},
$$

or equivalently in pure matrix-vector notation

$$
\tilde{\sigma}_{\alpha}=\sum_{\beta=1}^{6} \tilde{C}_{\alpha \beta} \tilde{\varepsilon}_{\beta} .
$$

The advantage of the normalized representation used here is that the eigenvalues and eigentensors of the elasticity tensor, defined by $\lambda \boldsymbol{e}=\mathbb{C}[\boldsymbol{e}]$, are identical with the corresponding quantities of the matrix $\tilde{C}_{\alpha \beta}$. This does not apply in the case of the non-normalized Voigt notation. 
In the case of a cubic crystal symmetry, the constitutive tensor $\mathbb{C}_{i j k l}$ of the elastic constants in the normalized Voigt notation is written as

$$
\tilde{C}_{\alpha \beta}=\left(\begin{array}{cccccc}
\tilde{C}_{11} & \tilde{C}_{12} & \tilde{C}_{12} & 0 & 0 & 0 \\
\tilde{C}_{12} & \tilde{C}_{11} & \tilde{C}_{12} & 0 & 0 & 0 \\
\tilde{C}_{12} & \tilde{C}_{12} & \tilde{C}_{11} & 0 & 0 & 0 \\
0 & 0 & 0 & \tilde{C}_{44} & 0 & 0 \\
0 & 0 & 0 & 0 & \tilde{C}_{44} & 0 \\
0 & 0 & 0 & 0 & 0 & \tilde{C}_{44}
\end{array}\right)
$$

where

$$
\tilde{C}_{11}=C_{11}, \quad \tilde{C}_{12}=C_{12}, \quad \tilde{C}_{44}=2 C_{44} .
$$

For the calculation of the invariants of a sixth-rank tensor, it is also useful to introduce a matrix representation. Since the tensor $\mathbb{D}$ constitutes energetically a quadratic form in the gradient of the elastic strain tensor and satisfies two minor and one major symmetry conditions, the orthonormal basis for symmetric second-rank tensors can be used to generate an 18-dimensional basis for $\mathbb{D}$. Based on the index functions

$$
f_{1}(\xi)=\{1,2,4,3,5,2,1,4,3,6,3,1,5,2,6,4,5,6\}
$$

and

$$
f_{2}(\xi)=\{1,1,2,1,3,2,2,1,2,3,3,3,1,3,2,3,2,1\}
$$

an 18-dimensional orthonormal tensor basis for sixth-rank tensors can be defined by

$$
\mathbb{F}_{\xi}=\boldsymbol{B}_{f_{1}(\xi)} \otimes \boldsymbol{e}_{f_{2}(\xi)}, \quad \xi=1, \ldots, 18,
$$

which satisfies

$$
\mathbb{F}_{\xi} \cdot \mathbb{F}_{\rho}=\delta_{\xi \rho}, \quad \xi, \rho=1, \ldots, 18 .
$$

Using this basis system, third-rank tensors in three-dimensional space are represented as first-rank tensors (vectors) in an 18-dimensional space. Analogously, sixth-rank tensors in three-dimensional space are represented as second-rank tensors in an 18-dimensional space.

The components of the double stress, the gradient of the elastic strain tensor and the constitutive tensor of the gradient-elastic constants are determined by projection onto the basis tensors

$$
\tilde{\tau}_{\xi}=\boldsymbol{\tau} \cdot \mathbb{F}_{\xi}, \quad \tilde{\gamma}_{\rho}=\nabla \boldsymbol{e} \cdot \mathbb{F}_{\rho}, \quad \tilde{D}_{\xi, \rho}=\mathbb{F}_{\xi} \cdot \mathbb{D}\left[\mathbb{F}_{\rho}\right] .
$$

The constitutive relation for the double stress tensor can be expressed by

$$
\boldsymbol{\tau}=\sum_{\xi=1}^{18} \tilde{\tau}_{\xi} \mathbb{F}_{\xi}=\sum_{\xi, \rho=1}^{18} \tilde{D}_{\xi, \rho} \tilde{\gamma}_{\rho} \mathbb{F}_{\xi},
$$

or equivalently in pure matrix-vector notation

$$
\tilde{\tau}_{\xi}=\sum_{\rho=1}^{18} \tilde{D}_{\xi, \rho} \tilde{\gamma}_{\rho} .
$$

The advantage of the normalized representation used here is that the eigenvalues and eigentensors of the constitutive tensor of gradient-elastic constants, which are defined by $\lambda \nabla \boldsymbol{e}=\mathbb{D}[\nabla \boldsymbol{e}]$, are identical with the corresponding quantities of the matrix $\tilde{D}_{\xi, \rho}$.

The $18 \times 18$ matrix representation of $\mathbb{D}$ has the form

$$
\tilde{D}_{\xi, \rho}=\left(\begin{array}{cccc}
\tilde{\mathbb{D}}_{1} & \mathbf{0} & \mathbf{0} & \mathbf{0} \\
\mathbf{0} & \tilde{\mathbb{D}}_{1} & \mathbf{0} & \mathbf{0} \\
\mathbf{0} & \mathbf{0} & \tilde{\mathbb{D}}_{1} & \mathbf{0} \\
\mathbf{0} & \mathbf{0} & \mathbf{0} & \tilde{\mathbb{D}}_{2}
\end{array}\right)
$$


with the symmetric $5 \times 5$ sub-matrix with 9 independent elements

$$
\tilde{\mathbb{D}}_{1}=\left(\begin{array}{ccccc}
\tilde{D}_{1,1} & \tilde{D}_{1,2} & \tilde{D}_{1,3} & \tilde{D}_{1,2} & \tilde{D}_{1,3} \\
\tilde{D}_{1,2} & \tilde{D}_{2,2} & \tilde{D}_{2,3} & \tilde{D}_{2,4} & \tilde{D}_{2,5} \\
\tilde{D}_{1,3} & \tilde{D}_{2,3} & \tilde{D}_{3,3} & \tilde{D}_{2,5} & \tilde{D}_{3,5} \\
\tilde{D}_{1,2} & \tilde{D}_{2,4} & \tilde{D}_{2,5} & \tilde{D}_{2,2} & \tilde{D}_{2,3} \\
\tilde{D}_{1,3} & \tilde{D}_{2,5} & \tilde{D}_{3,5} & \tilde{D}_{2,3} & \tilde{D}_{3,3}
\end{array}\right)
$$

and the symmetric $3 \times 3$ sub-matrix with 2 independent elements

$$
\tilde{\mathbb{D}}_{2}=\left(\begin{array}{ccc}
\tilde{D}_{16,16} & \tilde{D}_{16,17} & \tilde{D}_{16,17} \\
\tilde{D}_{16,17} & \tilde{D}_{16,16} & \tilde{D}_{16,17} \\
\tilde{D}_{16,17} & \tilde{D}_{16,17} & \tilde{D}_{16,16}
\end{array}\right)
$$

with the gradient-elastic constants in normalized Voigt notation to be given as

$$
\begin{aligned}
\tilde{D}_{1,1} & =2\left(a_{1}+a_{2}+a_{3}+a_{4}+a_{5}\right)+4\left(a_{6}+a_{7}+a_{10}\right)+a_{8}+2 a_{9}+a_{11} \\
\tilde{D}_{1,2} & =a_{1}+2 a_{2}+a_{9} \\
\tilde{D}_{1,3} & =\left(a_{1}+2 a_{3}+2 a_{10}\right) / \sqrt{2} \\
\tilde{D}_{2,2} & =2\left(a_{2}+a_{4}\right)+a_{8} \\
\tilde{D}_{2,3} & =\left(a_{1}+2 a_{5}+2 a_{7}\right) / \sqrt{2}, \\
\tilde{D}_{2,4} & =2 a_{2} \\
\tilde{D}_{2,5} & =a_{1} / \sqrt{2} \\
\tilde{D}_{3,3} & =a_{3}+2 a_{4}+a_{5}+2 a_{6}, \\
\tilde{D}_{3,5} & =a_{3} \\
\tilde{D}_{16,16} & =2 a_{4} \\
\tilde{D}_{16,17} & =a_{5} .
\end{aligned}
$$

Therefore, the constitutive tensor $\mathbb{D}$ in the normalized Voigt notation is represented by the symmetric block diagonal matrix (122), which is expressed in linear combinations of the 11 gradient-elastic constants through the relations (125a)-(125k) .

Comparing Eqs. (125a)-(125k) and (59a)-(59k), the relations between $\tilde{D}_{\xi, \rho}$ and $D_{\xi, \rho}$ read as

$$
\begin{aligned}
& \tilde{D}_{1,1}=D_{1,1}, \quad \tilde{D}_{1,2}=D_{1,2}, \quad \tilde{D}_{1,3}=\sqrt{2} D_{1,3}, \quad \tilde{D}_{2,2}=D_{2,2}, \quad \tilde{D}_{2,3}=\sqrt{2} D_{2,3}, \quad \tilde{D}_{2,4}=D_{2,4}, \\
& \tilde{D}_{2,5}=\sqrt{2} D_{2,5}, \quad \tilde{D}_{3,3}=2 D_{3,3}, \quad \tilde{D}_{3,5}=2 D_{3,5}, \quad \tilde{D}_{16,16}=2 D_{16,16}, \quad \tilde{D}_{16,17}=2 D_{16,17} .
\end{aligned}
$$

\subsection{Eigenvalues and positive definiteness of the constitutive tensors}

In this subsection, we derive the independent eigenvalues of the constitutive tensors $\mathbb{C}$ and $\mathbb{D}$ of first strain gradient elasticity for the cubic as well as for the isotropic case. The conditions for the positive definiteness of the two tensors are also given for both examined cases.

The matrix $\tilde{C}_{\alpha \beta}$ has 3 independent eigenvalues, which are given by

$$
\lambda_{1}^{C}=\tilde{C}_{11}+2 \tilde{C}_{12}, \quad \lambda_{2}^{C}=\tilde{C}_{11}-\tilde{C}_{12}, \quad \lambda_{3}^{C}=\tilde{C}_{44} .
$$

Here, $\lambda_{1}^{C}$ is a single eigenvalue, $\lambda_{2}^{C}$ is a double eigenvalue, and $\lambda_{3}^{C}$ is a triple eigenvalue. In the isotropic case $\lambda_{2}^{C}=\lambda_{3}^{C}$ and therefore only two independent eigenvalues exist.

Due to the specific matrix structure of $\tilde{D}_{\xi, \rho}$ given in Eq. (122), it follows that the eigenvalues of $\tilde{D}_{\xi, \rho}$ are equal to the eigenvalues of $\tilde{\mathbb{D}}_{1}$ and that ones of $\tilde{\mathbb{D}}_{2}$. The 18 eigenvalues of the tensor $\mathbb{D}$ consist of 5 independent 
Table 5 Eigenvalues of the sixth-rank constitutive tensor $\mathbb{D}$ for different cubic materials are given in units of [eV/Å]

\begin{tabular}{|c|c|c|c|c|c|}
\hline & $\mathrm{Al}(\mathrm{fcc})$ & $\mathrm{Cu}(\mathrm{fcc})$ & $\mathrm{Fe}(\mathrm{bcc})$ & W (bcc) & Multiplicity \\
\hline$\lambda_{1}^{D}$ & 0.03560 & 0.00005 & 0.06598 & 0.48426 & 2 \\
\hline$\lambda_{2}^{D}$ & 0.93596 & 0.22441 & 2.36399 & 4.18264 & 1 \\
\hline$\lambda_{3}^{D}$ & 0.87576 & 0.83490 & 0.65338 & 1.94819 & 3 \\
\hline$\lambda_{4}^{D}$ & 0.04980 & 0.04664 & -0.48601 & -0.94259 & 3 \\
\hline$\lambda_{5}^{D}$ & 1.70599 & 1.70391 & 2.47144 & 3.90703 & 3 \\
\hline$\lambda_{6}^{D}$ & 1.06408 & 0.07599 & 1.03077 & 3.18388 & 3 \\
\hline$\lambda_{7}^{D}$ & 0.17883 & 0.04664 & 0.77479 & 0.85833 & 3 \\
\hline
\end{tabular}

eigenvalues of the $5 \times 5$ sub-matrix $\tilde{\mathbb{D}}_{2}$, which appears three times, and the 2 independent eigenvalues of the $3 \times 3$ sub-matrix $\tilde{\mathbb{D}}_{1}$, from which one has multiplicity 2 . That is, the tensor $\mathbb{D}$ has 7 independent eigenvalues.

Specifically, the two independent eigenvalues of $\tilde{\mathbb{D}}_{2}$ are given by

$$
\lambda_{1}^{D}=-\tilde{D}_{16,17}+\tilde{D}_{16,16}
$$

with multiplicity two, and

$$
\lambda_{2}^{D}=2 \tilde{D}_{16,17}+\tilde{D}_{16,16}
$$

with multiplicity one.

The 5 independent eigenvalues of $\tilde{\mathbb{D}}_{1}$ with multiplicity one are given by

$$
\lambda_{3,4}^{D}=\left(k_{1} \pm \sqrt{k_{2}-4 k_{3}}\right) / 2
$$

with

$$
\begin{aligned}
& k_{1}=\tilde{D}_{2,2}-\tilde{D}_{2,4}+\tilde{D}_{3,3}-\tilde{D}_{3,5}, \\
& k_{2}=\left(-\tilde{D}_{2,2}+\tilde{D}_{2,4}-\tilde{D}_{3,3}+\tilde{D}_{3,5}\right)^{2}, \\
& k_{3}=-\tilde{D}_{2,3}^{2}+2 \tilde{D}_{2,3} \tilde{D}_{2,5}-\tilde{D}_{2,5}^{2}+\tilde{D}_{2,2} \tilde{D}_{3,3}-\tilde{D}_{2,4} \tilde{D}_{3,3}-\tilde{D}_{2,2} \tilde{D}_{3,5}+\tilde{D}_{2,4} \tilde{D}_{3,5},
\end{aligned}
$$

and, additionally, with the roots $\lambda_{5}^{D}, \lambda_{6}^{D}, \lambda_{7}^{D}$ of the cubic algebraic equation

$$
0=k_{4}+k_{5} x+k_{6} x^{2}+x^{3}
$$

with the coefficients

$$
\begin{aligned}
k_{4}= & 2 \tilde{D}_{1,3}^{2} \tilde{D}_{2,2}-4 \tilde{D}_{1,2} \tilde{D}_{1,3} \tilde{D}_{2,3}+\tilde{D}_{1,1} \tilde{D}_{2,3}^{2}+\tilde{D} \tilde{D}_{1,3}^{2} \tilde{D}_{2,4} \\
& -4 \tilde{D}_{1,2} \tilde{D}_{1,3} \tilde{D}_{2,5}+2 \tilde{D}_{1,1} \tilde{D}_{2,3} \tilde{D}_{2,5}+\tilde{D}_{1,1} \tilde{D}_{2,5}^{2}+2 \tilde{D}_{1,2}^{2} \tilde{D}_{3,3} \\
& -\tilde{D}_{1,1} \tilde{D}_{2,2} \tilde{D}_{3,3}-\tilde{D}_{1,1} \tilde{D}_{2,4} \tilde{D}_{3,3}+2 \tilde{D}_{1,2}^{2} \tilde{D}_{3,5}-\tilde{D}_{1,1} \tilde{D}_{2,2} \tilde{D}_{3,5}-\tilde{D}_{1,1} \tilde{D}_{2,4} \tilde{D}_{35}, \\
k_{5}= & -2 \tilde{D}_{1,2}^{2}-2 \tilde{D}_{1,3}^{2}+\tilde{D}_{1,1} \tilde{D}_{2,2}-\tilde{D}_{2,3}^{2}+\tilde{D}_{1,1} \tilde{D}_{2,4}-2 \tilde{D}_{2,3} \tilde{D}_{2,5}-\tilde{D}_{2,5}^{2} \\
& +\tilde{D}_{1,1} \tilde{D}_{3,3}+\tilde{D}_{2,2} \tilde{D}_{3,3}+\tilde{D}_{2,4} \tilde{D}_{3,3}+\tilde{D}_{1,1} \tilde{D}_{3,5}+\tilde{D}_{2,2} \tilde{D}_{3,5}+\tilde{D}_{2,4} \tilde{D}_{3,5}, \\
k_{6}= & -\left(\tilde{D}_{1,1}+\tilde{D}_{2,2}+\tilde{D}_{2,4}+\tilde{D}_{3,3}+\tilde{D}_{3,5}\right) .
\end{aligned}
$$

In Table 5, the numerical values of the eigenvalues of the constitutive tensor $\mathbb{D}_{i j m k l n}$ for the four considered materials are given in units of $[\mathrm{eV} / \AA]$. One can see that in the sense of $\mathbb{D}$, each of the five distinct eigenvalues of $\tilde{\mathbb{D}}_{1}$ has the multiplicity of three. Hence, $\mathbb{D}$ has one eigenvalue of multiplicity of one, one of multiplicity of two and five eigenvalues of multiplicity of three. 
Concerning positive definiteness, a matrix is positive definite if and only if all its eigenvalues are positive. Therefore, the values of the elastic constants $C_{11}, C_{12}$ and $C_{44}$ have to obey the following 3 conditions of positive definiteness of the tensor $\mathbb{C}$ :

$$
\begin{aligned}
& \lambda_{1}^{C}=C_{11}+2 C_{12}>0, \\
& \lambda_{2}^{C}=C_{11}-C_{12}>0, \\
& \lambda_{3}^{C}=2 C_{44}>0 .
\end{aligned}
$$

The conditions of positive definiteness for the gradient-elastic constants are given by the positive values of the 7 independent eigenvalues of $\mathbb{D}$ as follows

$$
\lambda_{\alpha}^{D}>0, \quad \alpha=1, \ldots, 7
$$

In Table 5, it can be seen that the conditions of positive definiteness are fulfilled for $\mathrm{Al}$ and $\mathrm{Cu}$ but not for Fe and $\mathrm{W}$, since one eigenvalue is negative, $\lambda_{4}^{D}<0$. This result is in agreement with the result obtained in Sect. 3.3 based on the Sylvester criterion.

In the isotropic first strain gradient elasticity, $H=0$, that is, $\lambda_{2}^{C}=\lambda_{3}^{C}$, and hence, the constitutive tensor $\mathbb{C}$ has only two independent eigenvalues, namely

$$
\begin{aligned}
& \lambda_{1}^{C}=C_{11}+2 C_{12}, \\
& \lambda_{2}^{C}=2 C_{44} .
\end{aligned}
$$

The eigenvalue $\lambda_{1}^{C}$ has multiplicity one, and $\lambda_{2}^{C}$ has multiplicity five. Therefore, there exist only two independent conditions for the positive definiteness of the tensor $\mathbb{C}$.

In the isotropic case, the matrices $\tilde{\mathbb{D}}_{1}$ and $\tilde{\mathbb{D}}_{2}$ take the form

$$
\tilde{\mathbb{D}}_{1}=\left(\begin{array}{ccccc}
2\left(a_{1}+a_{2}+a_{3}+a_{4}+a_{5}\right) & a_{1}+2 a_{2} & \sqrt{2}\left(a_{1} / 2+a_{3}\right) & a_{1}+2 a_{2} & \sqrt{2}\left(a_{1} / 2+a_{3}\right) \\
a_{1}+2 a_{2} & 2\left(a_{2}+a_{4}\right) & \sqrt{2}\left(a_{1} / 2+a_{5}\right) & 2 a_{2} & a_{1} / \sqrt{2} \\
\sqrt{2}\left(a_{1} / 2+a_{3}\right) & \sqrt{2}\left(a_{1} / 2+a_{5}\right) & a_{3}+2 a_{4}+a_{5} & a_{1} / \sqrt{2} & a_{3} \\
a_{1}+2 a_{2} & 2 a_{2} & a_{1} / \sqrt{2} & 2\left(a_{2}+a_{4}\right) & \sqrt{2}\left(a_{1} / 2+a_{5}\right) \\
\sqrt{2}\left(a_{1} / 2+a_{3}\right) & a_{1} / \sqrt{2} & a_{3} & \sqrt{2}\left(a_{1} / 2+a_{5}\right) & a_{3}+2 a_{4}+a_{5}
\end{array}\right)
$$

and

$$
\tilde{\mathbb{D}}_{2}=\left(\begin{array}{ccc}
2 a_{4} & a_{5} & a_{5} \\
a_{5} & 2 a_{4} & a_{5} \\
a_{5} & a_{5} & 2 a_{4}
\end{array}\right)
$$

The matrix $\tilde{\mathbb{D}}_{2}$ has 2 independent eigenvalues

$$
\begin{aligned}
& \lambda_{1}^{D}=2 a_{4}-a_{5}, \\
& \lambda_{2}^{D}=2\left(a_{4}+a_{5}\right),
\end{aligned}
$$

where $\lambda_{1}^{D}$ has multiplicity two and $\lambda_{2}^{D}$ has multiplicity one. The matrix $\tilde{\mathbb{D}}_{1}$ has 4 independent eigenvalues, namely the $\lambda_{1}^{D}$ with multiplicity one and $\lambda_{2}^{D}$ with multiplicity two, and the eigenvalues

$$
\begin{aligned}
& \lambda_{3}^{D}=\frac{1}{2}\left(2 a_{1}+6 a_{2}+4 a_{3}+4 a_{4}+a_{5}-\sqrt{k_{7}}\right), \\
& \lambda_{4}^{D}=\frac{1}{2}\left(2 a_{1}+6 a_{2}+4 a_{3}+4 a_{4}+a_{5}+\sqrt{k_{7}}\right),
\end{aligned}
$$

where

$$
k_{7}=24 a_{1}^{2}+24 a_{1} a_{2}+16 a_{1} a_{3}+28 a_{1} a_{5}+36 a_{2}^{2}-32 a_{2} a_{3}+4 a_{2} a_{5}+16 a_{3}^{2}+16 a_{3} a_{5}+9 a_{5}^{2}
$$

with multiplicity one. Here, a new numbering of the eigenvalues is used. 
Therefore, the tensor $\mathbb{D}$ has four independent eigenvalues given in terms of the gradient-elastic constants $a_{1}, \ldots, a_{5}$. These eigenvalues provide 4 conditions for the positive definiteness of $\mathbb{D}$ in isotropic first strain gradient elasticity. For the investigation of positive definiteness in isotropic first strain gradient elasticity, the Sylvester criterion is commonly used in the literature leading to more than the necessary conditions and also to different conditions mostly due to different used basis. However, only the normalized Voigt notation gives the correct independent eigenvalues of the constitutive tensors and consequently the correct number of the necessary and sufficient conditions for the positive definiteness.

\section{Voigt-type average of the sixth-rank constitutive tensor $\mathbb{D}$}

In Sect. 3, we have seen that there are cubic materials with centrosymmetry such as $\mathrm{Al}$ and $\mathrm{W}$ which are nearly isotropic ${ }^{4}$ with respect to the fourth-rank constitutive tensor $\mathbb{C}_{i j k l}$, whereas they are not isotropic with respect to the constitutive tensor $\mathbb{D}_{i j m k l n}$, which does not satisfy any of the six isotropy conditions (67). For that reason, we use here a Voigt-type averaging for the sixth-rank constitutive tensor $\mathbb{D}_{i j m k l n}$ in order to compute isotropic gradient-elastic constants.

For the calculation of the Voigt-type average of the sixth-rank constitutive tensor $\mathbb{D}_{i j m k l n}$, the tensor $\mathbb{D}_{i j m k l n}$ must be averaged over all orientations. For complete random orientation of an isotopic material, such linear averages can be calculated from the following condition: The linear invariants of the sixth-rank constitutive tensor representing the single crystal of cubic type must be equal to the linear invariants of the corresponding averaged isotropic sixth-rank constitutive tensor. Such a method was first used by Leibfried [36] (see also $[18,26])$ to calculate the Voigt average of the second-order elastic constants for cubic symmetry, and by Bross [7] (see also [6]) to calculate the Voigt average of the first kind of the third-order elastic constants for cubic symmetry. In this work, we generalize the Voigt average for the sixth-rank constitutive tensor $\mathbb{D}_{i j m k l n}$ in order to calculate the Voigt-type average of the gradient-elastic constants in Toupin-Mindlin anisotropic first strain gradient elasticity for cubic materials with centrosymmetry.

Remark 1 The obtained averaged isotropic gradient-elastic constants defined in this manner are valid for constant double strain. This is an important constraint that prevents the Voigt-type average for both constitutive tensors, $\mathbb{C}_{i j k l}$ and $\mathbb{D}_{i j m k l n}$, in the framework of anisotropic first strain gradient elasticity, since for the Voigt average of the constitutive tensor $\mathbb{C}_{i j k l}$, the constraint of constant elastic strain would lead to zero double strain.

Remark 2 It should be noted that commonly the term Voigt average is related to a strict upper bound of the strain energy density or the dissipation potential for linear and nonlinear materials, which depends generally only on the one-point statistics of microstructure (see, e.g., [8]). Such an interpretation is not applied here.

The sixth-rank constitutive tensor of an averaged isotropic crystal, $\overline{\mathbb{D}}_{i j m k l n}$, is given by

$$
\begin{aligned}
\overline{\mathbb{D}}_{i j m k l n}= & \frac{\bar{a}_{1}}{2}\left(\delta_{i j} \delta_{k m} \delta_{l n}+\delta_{i j} \delta_{k n} \delta_{l m}+\delta_{k l} \delta_{i m} \delta_{j n}+\delta_{k l} \delta_{i n} \delta_{j m}\right)+2 \bar{a}_{2} \delta_{i j} \delta_{k l} \delta_{m n} \\
& +\frac{\bar{a}_{3}}{2}\left(\delta_{j k} \delta_{i m} \delta_{l n}+\delta_{i k} \delta_{j m} \delta_{l n}+\delta_{i l} \delta_{j m} \delta_{k n}+\delta_{j l} \delta_{i m} \delta_{k n}\right)+\bar{a}_{4}\left(\delta_{i l} \delta_{j k} \delta_{m n}+\delta_{i k} \delta_{j l} \delta_{m n}\right) \\
& +\frac{\bar{a}_{5}}{2}\left(\delta_{j k} \delta_{i n} \delta_{l m}+\delta_{i k} \delta_{j n} \delta_{l m}+\delta_{j l} \delta_{k m} \delta_{i n}+\delta_{i l} \delta_{k m} \delta_{j n}\right),
\end{aligned}
$$

where $\bar{a}_{1}, \bar{a}_{2}, \bar{a}_{3}, \bar{a}_{4}, \bar{a}_{5}$ are the five averaged isotropic gradient-elastic constants.

The five linear invariants of the sixth-rank constitutive tensor $\mathbb{D}_{i j m k l n}$ are

$$
\begin{aligned}
I_{1}^{\mathbb{D}} & =\mathbb{D}_{i i j j k k}, \\
I_{2}^{\mathbb{D}} & =\mathbb{D}_{i i j k k j,}, \\
I_{3}^{\mathbb{D}} & =\mathbb{D}_{i j i j k k}, \\
I_{4}^{\mathbb{D}} & =\mathbb{D}_{i j k i j k}, \\
I_{5}^{\mathbb{D}} & =\mathbb{D}_{i j k j k i} .
\end{aligned}
$$

\footnotetext{
${ }^{4}$ Diamond is also a nearly isotropic material.
} 
From the condition that the five linear invariants of the constitutive tensor of sixth-rank $\mathbb{D}_{i j m k l n}$ (representing the single crystal of cubic type) must be equal to the ones of the sixth-rank tensor $\overline{\mathbb{D}}_{i j m k l n}$ of the averaged isotropic crystal, we obtain the following relations:

$$
\begin{aligned}
& I_{1}^{\mathbb{D}}=I_{1}^{\overline{\mathbb{D}},} \\
& I_{2}^{\mathbb{D}}=I_{2}^{\overline{\mathbb{D}},} \\
& I_{3}^{\mathbb{D}}=I_{3}^{\overline{\mathbb{D}},} \\
& I_{4}^{\mathbb{D}}=I_{4}^{\overline{\mathbb{D}}}, \\
& I_{5}^{\mathbb{D}}=I_{5}^{\mathbb{D}} .
\end{aligned}
$$

Substituting Eqs. (40) and (154) into Eqs. (156a)-(156e), we obtain the following system of equations:

$$
\begin{aligned}
& 21 a_{1}+18 a_{2}+12 a_{3}+6 a_{4}+12 a_{5}+12 a_{6}+18 a_{7}+3 a_{8}+12 a_{9}+18 a_{10}+3 a_{11} \\
& =21 \bar{a}_{1}+18 \bar{a}_{2}+12 \bar{a}_{3}+6 \bar{a}_{4}+12 \bar{a}_{5} \\
& 18 a_{1}+54 a_{2}+6 a_{3}+18 a_{4}+6 a_{5}+12 a_{6}+12 a_{7}+9 a_{8}+18 a_{9}+12 a_{10}+3 a_{11} \\
& =18 \bar{a}_{1}+54 \bar{a}_{2}+6 \bar{a}_{3}+18 \bar{a}_{4}+6 \bar{a}_{5} \\
& 12 a_{1}+6 a_{2}+24 a_{3}+12 a_{4}+9 a_{5}+18 a_{6}+12 a_{7}+3 a_{8}+6 a_{9}+24 a_{10}+3 a_{11} \\
& =12 \bar{a}_{1}+6 \bar{a}_{2}+24 \bar{a}_{3}+12 \bar{a}_{4}+9 \bar{a}_{5} \\
& 6 a_{1}+18 a_{2}+12 a_{3}+36 a_{4}+12 a_{5}+24 a_{6}+12 a_{7}+9 a_{8}+6 a_{9}+12 a_{10}+3 a_{11} \\
& =6 \bar{a}_{1}+18 \bar{a}_{2}+12 \bar{a}_{3}+36 \bar{a}_{4}+12 \bar{a}_{5} \\
& 12 a_{1}+6 a_{2}+9 a_{3}+12 a_{4}+24 a_{5}+18 a_{6}+24 a_{7}+3 a_{8}+6 a_{9}+12 a_{10}+3 a_{11} \\
& =12 \bar{a}_{1}+6 \bar{a}_{2}+9 \bar{a}_{3}+12 \bar{a}_{4}+24 \bar{a}_{5} .
\end{aligned}
$$

Solving the above system for $\bar{a}_{1}, \bar{a}_{2}, \bar{a}_{3}, \bar{a}_{4}$, and $\bar{a}_{5}$, we obtain the five Voigt-type averaged isotropic gradientelastic constants:

$$
\begin{aligned}
& \bar{a}_{1}=a_{1}+\frac{2}{5}\left(a_{7}+a_{9}+a_{10}\right)+\frac{2}{35} a_{11}, \\
& \bar{a}_{2}=a_{2}+\frac{1}{10}\left(a_{8}+2 a_{9}\right)+\frac{1}{70} a_{11}, \\
& \bar{a}_{3}=a_{3}+\frac{2}{5}\left(a_{6}+2 a_{10}\right)+\frac{2}{35} a_{11}, \\
& \bar{a}_{4}=a_{4}+\frac{1}{5}\left(2 a_{6}+a_{8}\right)+\frac{1}{35} a_{11}, \\
& \bar{a}_{5}=a_{5}+\frac{2}{5}\left(a_{6}+2 a_{7}\right)+\frac{2}{35} a_{11} .
\end{aligned}
$$

In terms of the Voigt-type averaged isotropic gradient-elastic constants, the corresponding two characteristic lengths are given by

$$
\begin{aligned}
& \bar{\ell}_{1}^{2}=\frac{2\left(\bar{a}_{1}+\bar{a}_{2}+\bar{a}_{3}+\bar{a}_{4}+\bar{a}_{5}\right)}{\lambda+2 \mu}, \\
& \bar{\ell}_{2}^{2}=\frac{\bar{a}_{3}+2 \bar{a}_{4}+\bar{a}_{5}}{2 \mu},
\end{aligned}
$$

where $\mu=C_{44}$ and $\lambda=C_{12}$. Notice that the mathematical structure of $\bar{\ell}_{1}^{2}$ and $\bar{\ell}_{2}^{2}$ is the same as those of $\ell_{1}^{2}$ and $\ell_{2}^{2}$ [see Eqs. (78) and (79)].

For example, for aluminum and tungsten, using Eqs. (158a)-(158e) together with the values of the gradientelastic constants from Table 2 and the values of $C_{44}$ and $C_{12}$ from Table 1, we compute the five Voigt-type averaged isotropic gradient-elastic constants and the corresponding characteristic lengths, which are given in Table 6. It can be seen that for both $\mathrm{Al}$ and $\mathrm{W}$, it yields: $\ell_{1} / a \approx 0.3$ and $\ell_{2} / a \approx 0.3$. An important remark here is that the conditions of positive definiteness for the constitutive tensor of the gradient-elastic constants, 
Table 6 Voigt-type averaged isotropic gradient-elastic constants and corresponding characteristic lengths for aluminum and tungsten

\begin{tabular}{lcr}
\hline & $\mathrm{Al}(\mathrm{fcc})$ & $\mathrm{W}(\mathrm{bcc})$ \\
\hline $\bar{a}_{1}[\mathrm{eV} / \AA]$ & -0.13862 & 0.02387 \\
$\bar{a}_{2}[\mathrm{eV} / \AA]$ & 0.22500 & 0.19215 \\
$\bar{a}_{3}[\mathrm{eV} / \AA]$ & 0.10877 & 0.43264 \\
$\bar{a}_{4}[\mathrm{eV} / \AA]$ & 0.15309 & 0.54907 \\
$\bar{a}_{5}[\mathrm{eV} / \AA]$ & 0.21632 & 0.28799 \\
$\bar{\ell}_{1}[\AA]$ & 1.20272 & 0.94654 \\
$\bar{\ell}_{2}[\AA]$ & 1.26566 & 0.94509 \\
$\bar{\ell}_{1} / a$ & 0.2970 & 0.2990 \\
$\bar{\ell}_{2} / a$ & 0.3125 & 0.2986 \\
\hline
\end{tabular}

Eqs. (A.7a)-(A.7e), are fulfilled for aluminum (Al) and tungsten (W) using the Voigt-type averaged isotropic gradient-elastic constants from Table 6 and Eqs. (82a)-(82k). In the same results, we conclude if we use the conditions of positive definiteness with the eigenvalue method. Indeed, the eigenvalues given by Eqs. (149)(152) are positive for the values of the Voigt-type averaged isotropic gradient-elastic constants for aluminum (Al) and tungsten $(\mathrm{W})$ given in Table 6.

\section{Conclusions}

Comparisons between anisotropic and isotropic first strain gradient elasticity for cubic materials with centrosymmetry of point group $m \overline{3} m$ as well as comparisons between classical elasticity and first strain gradient elasticity are remarkable. From the presented results, the following favorable conclusions can be reached (see also Table 7):

- There are 3 characteristic lengths appearing in the 3 modified Helmholtz operators, which are part of the Mindlin operator, in the Toupin-Mindlin anisotropic first strain gradient elasticity for cubic materials with centrosymmetry.

- There are 3 characteristic lengths for 3 elastic constants $C_{11}, C_{12}$ and $C_{44}$ for cubic crystals with centrosymmetry in anisotropic first strain gradient elasticity, whereas there are 2 characteristic lengths for 2 elastic constants (Lamé constants) in isotropic first strain gradient elasticity.

- There are 11 gradient-elastic constants in the Toupin-Mindlin anisotropic first strain gradient elasticity for cubic materials with centrosymmetry, whereas there are 5 gradient-elastic constants in the isotropic version.

- In the Toupin-Mindlin anisotropic first strain gradient elasticity for cubic materials with centrosymmetry, the necessary and sufficient conditions for the positive definiteness of the strain energy density conclude to 3 conditions (inequalities) for the elastic constants and 7 conditions for the gradient-elastic constants. We conclude in this result with two different but equivalent methods: one with the Sylvester criterion based on a Voigt-matrix representation and another one with the eigenvalue method based on the normalized Voigt-matrix representation. It should be noted that the matrix representation of the constitutive tensors in the Voigt notation yields non-tensorial representations since non-normalized basis systems are used.

- There is 1 isotropy condition for the constitutive tensor $\mathbb{C}_{i j k l}$ (as in classical elasticity) and 6 isotropy conditions for the constitutive tensor $\mathbb{D}_{i j m k l n}$, that is 7 isotropy conditions in total, in the Toupin-Mindlin anisotropic first strain gradient elasticity for cubic materials with centrosymmetry.

- From the physical point of view, it is reasonable that in the framework of classical elasticity, one can obtain a nearly isotropic material from a cubic material, since it exists only the constitutive tensor of the elastic constants, whereas in the framework of first strain gradient elasticity, which is valid at small scales where the microstructure is dominant, the anisotropic behavior resists due to the appearance of the constitutive tensor of higher rank, preventing the fulfillment of the corresponding isotropy conditions.

- Aluminum and tungsten are nearly isotropic with respect to the fourth-rank constitutive tensor $\mathbb{C}_{i j k l}$, whereas none of the four considered cubic crystals $(\mathrm{Al}, \mathrm{Cu}, \mathrm{Fe}, \mathrm{W})$ is isotropic with respect to the sixthrank constitutive tensor $\mathbb{D}_{i j m k l n}$.

- There are 5 lattice relations for the gradient-elastic constants in a lattice-theoretical approach of anisotropic first strain gradient elasticity for cubic materials with centrosymmetry leading to only 6 independent 
Table 7 Independent components of the elastic and gradient-elastic constants

\begin{tabular}{lll}
\hline Elastic and gradient-elastic constants & $\mathbb{C}_{i j k l}$ & $\mathbb{D}_{i j m k l n}$ \\
\hline Independent components_cubic & 3 & 11 \\
Independent components_isotropic & 2 & 5 \\
Isotropy conditions & 1 & 6 \\
Independent components due to (lattice relations)_cubic & - & $6(5)$ \\
Independent components due to (lattice relations)_isotropic & - & $2(3)$ \\
Independent components due to (Cauchy relations)_cubic & $2(1)$ & $3(8)$ \\
\hline
\end{tabular}

gradient-elastic constants instead of 11 ones. In the isotropic limit, there are 3 lattice relations for the gradient-elastic constants leading to 2 independent gradient-elastic constants instead of 5 ones.

- In the Toupin-Mindlin anisotropic first strain gradient elasticity for cubic materials with centrosymmetry, there are 8 generalized Cauchy relations for the gradient-elastic constants in addition to 1 Cauchy relation for the elastic constants (as in classical elasticity).

- The independent eigenvalues of the constitutive tensors $\mathbb{C}$ and $\mathbb{D}$ of first strain gradient elasticity have been derived based on the normalized Voigt notation. For cubic materials with centrosymmetry, the constitutive tensors $\mathbb{C}$ and $\mathbb{D}$ have 3 and 7 independent eigenvalues, respectively. In the isotropic case, $\mathbb{C}$ and $\mathbb{D}$ have 2 and 4 independent eigenvalues, respectively.

- A normalized Voigt notation is used for the representation of the constitutive relations between the field tensors (elastic strain tensor and gradient of the elastic strain tensor) and excitation field tensors (Cauchy stress tensor and double stress tensor). The advantage of the normalized Voigt notation is that the positive definiteness can be naturally related to the eigenvalues of the matrix representation of the constitutive tensors $\mathbb{C}$ and $\mathbb{D}$.

An important result of our computation is the 11 gradient-elastic constants and the 3 characteristic lengths for cubic materials with centrosymmetry given in Tables 2 and 3 as well as the 5 Voigt-type averaged isotropic gradient-elastic constants and the related 2 characteristic lengths given in Table 6 . They can be applied in the modeling of nanomechanical problems with a solid basis of material parameters of the Toupin-Mindlin gradient elasticity theory without any ad hoc assumption. Important applications of the material parameters are nanomechanical benchmark problems, eigenstrain and fracture problems in gradient elasticity, nanomechanical engineering and industrial problems, nanodevices as well as the modeling of non-singular dislocations leading to Ångström-mechanics of dislocations [33].

Acknowledgements Markus Lazar and Eleni Agiasofitou gratefully acknowledge the grants from the Deutsche Forschungsgemeinschaft (Grant numbers LA1974/4-2 and AG322/1-2). Markus Lazar would like to thank Giacomo Po for several clarifying remarks and interesting discussion.

Funding Open Access funding enabled and organized by Projekt DEAL.

Open Access This article is licensed under a Creative Commons Attribution 4.0 International License, which permits use, sharing, adaptation, distribution and reproduction in any medium or format, as long as you give appropriate credit to the original author(s) and the source, provide a link to the Creative Commons licence, and indicate if changes were made. The images or other third party material in this article are included in the article's Creative Commons licence, unless indicated otherwise in a credit line to the material. If material is not included in the article's Creative Commons licence and your intended use is not permitted by statutory regulation or exceeds the permitted use, you will need to obtain permission directly from the copyright holder. To view a copy of this licence, visit http://creativecommons.org/licenses/by/4.0/.

\title{
A Positive definiteness of $C_{\alpha \beta}$ and $D_{\xi, \rho}$ using the Sylvester criterion
}

\author{
A.1 Constitutive tensor $\mathbb{C}_{i j k l}: C_{\alpha \beta}$
}

Using the Voigt notation, the fourth-rank constitutive tensor $\mathbb{C}_{i j k l}$ for cubic crystals is contracted to the symmetric $6 \times 6$ matrix, $C_{\alpha \beta}, \alpha, \beta=1, \ldots, 6$ in a reference system whose axes coincide with the axes of the 
cubic crystal (see, e.g., [44]):

$$
C_{\alpha \beta}=\left(\begin{array}{cccccc}
C_{11} & C_{12} & C_{12} & 0 & 0 & 0 \\
C_{12} & C_{11} & C_{12} & 0 & 0 & 0 \\
C_{12} & C_{12} & C_{11} & 0 & 0 & 0 \\
0 & 0 & 0 & C_{44} & 0 & 0 \\
0 & 0 & 0 & 0 & C_{44} & 0 \\
0 & 0 & 0 & 0 & 0 & C_{44}
\end{array}\right)
$$

According to Sylvester's criterion: "A real, symmetric matrix is positive definite if and only if all its (leading) principal minors are positive" $[10,15,54]$. This leads to the following inequalities that have to be satisfied by the elastic constants

$$
C_{11}>0, \quad C_{11}-C_{12}>0, \quad C_{11}+2 C_{12}>0, \quad C_{44}>0 .
$$

The first condition is implied by the second and third conditions. Therefore, the values of the elastic constants $C_{11}, C_{12}$ and $C_{44}$ have to obey the following 3 conditions of positive definiteness of the matrix $C_{\alpha \beta}$

$$
C_{11}-C_{12}>0, \quad C_{11}+2 C_{12}>0, \quad C_{44}>0 .
$$

\section{A. 2 Constitutive tensor $\mathbb{D}_{i j m k l n}: D_{\xi, \rho}$}

Using the Voigt notation, the sixth-rank constitutive tensor $\mathbb{D}_{i j m k l n}$ for cubic crystals is contracted to the following block-diagonal symmetric $18 \times 18$ matrix, $D_{\xi, \rho}, \xi, \rho=1, \ldots, 18$, in a reference system whose axes coincide with the axes of the cubic crystal (see, e.g., $[1,4])$ :

$$
D_{\xi, \rho}=\left(\begin{array}{cccc}
\boldsymbol{A}_{(9)} & \mathbf{0} & \mathbf{0} & \mathbf{0} \\
\mathbf{0} & \boldsymbol{A}_{(9)} & \mathbf{0} & \mathbf{0} \\
\mathbf{0} & \mathbf{0} & \boldsymbol{A}_{(9)} & \mathbf{0} \\
\mathbf{0} & \mathbf{0} & \mathbf{0} & \boldsymbol{J}_{(2)}
\end{array}\right)
$$

with the symmetric $5 \times 5$ sub-matrix with 9 independent material parameters

$$
\boldsymbol{A}_{(9)}=\left(\begin{array}{lllll}
D_{1,1} & D_{1,2} & D_{1,3} & D_{1,2} & D_{1,3} \\
D_{1,2} & D_{2,2} & D_{2,3} & D_{2,4} & D_{2,5} \\
D_{1,3} & D_{2,3} & D_{3,3} & D_{2,5} & D_{3,5} \\
D_{1,2} & D_{2,4} & D_{2,5} & D_{2,2} & D_{2,3} \\
D_{1,3} & D_{2,5} & D_{3,5} & D_{2,3} & D_{3,3}
\end{array}\right)
$$

and the symmetric $3 \times 3$ sub-matrix with 2 independent material parameters

$$
\boldsymbol{J}_{(2)}=\left(\begin{array}{lll}
D_{16,16} & D_{16,17} & D_{16,17} \\
D_{16,17} & D_{16,16} & D_{16,17} \\
D_{16,17} & D_{16,17} & D_{16,16}
\end{array}\right) .
$$


The conditions of positive definiteness of the symmetric matrix $D$ (Sylvester's criterion) lead to the following inequalities:

$$
\begin{aligned}
& D_{1,1}>0, \quad D_{1,1} D_{2,2}-D_{1,2}^{2}>0 \\
& D_{1,1} D_{2,2} D_{3,3}-D_{1,1} D_{2,3}^{2}+2 D_{1,2} D_{1,3} D_{2,3}-D_{1,2}^{2} D_{3,3}-D_{1,3}^{2} D_{2,2}>0 \\
& D_{1,1} D_{2,2}^{2} D_{3,3}-D_{1,1} D_{2,2} D_{2,3}^{2}-D_{1,1} D_{2,2} D_{2,5}^{2}+2 D_{1,1} D_{2,3} D_{2,4} D_{2,5}-D_{1,1} D_{2,4}^{2} D_{3,3} \\
& \quad-2 D_{1,2}^{2} D_{2,2} D_{3,3}+D_{1,2}^{2} D_{2,3}^{2}-2 D_{1,2}^{2} D_{2,3} D_{2,5}+2 D_{1,2}^{2} D_{2,4} D_{3,3}+D_{1,2}^{2} D_{2,5}^{2} \\
& \quad+2 D_{1,2} D_{1,3} D_{2,2} D_{2,3}+2 D_{1,2} D_{1,3} D_{2,2} D_{2,5}-2 D_{1,2} D_{1,3} D_{2,3} D_{2,4}-2 D_{1,2} D_{1,3} D_{2,4} D_{2,5} \\
& \quad-D_{1,3}^{2} D_{2,2}^{2}+D_{1,3}^{2} D_{2,4}^{2}>0 \\
& \left(D_{2,2} D_{3,3}-D_{2,2} D_{3,5}-D_{2,3}^{2}+2 D_{2,3} D_{2,5}-D_{2,4} D_{3,3}+D_{2,4} D_{3,5}-D_{2,5}^{2}\right) \\
& \left(D_{1,1} D_{2,2} D_{3,3}+D_{1,1} D_{2,2} D_{3,5}-D_{1,1} D_{2,3}^{2}-2 D_{1,1} D_{2,3} D_{2,5}+D_{1,1} D_{2,4} D_{3,3}+D_{1,1} D_{2,4} D_{3,5}\right. \\
& \left.\quad-D_{1,1} D_{2,5}^{2}-2 D_{1,2}^{2} D_{3,3}-2 D_{1,2}^{2} D_{3,5}+4 D_{1,2} D_{1,3} D_{2,3}+4 D_{1,2} D_{1,3} D_{2,5}-2 D_{1,3}^{2} D_{2,2}-2 D_{1,3}^{2} D_{2,4}\right)>0 \\
& D_{16,16}-D_{16,17}>0,
\end{aligned}
$$

Note that $D_{16,16}>0$ is implied by the two conditions of (A.7e). Therefore, the conditions of positive definiteness for the gradient-elastic constants reduce to 7 conditions.

\section{References}

1. Admal, N.C., Marian, J., Po, G.: The atomistic representation of first strain-gradient elastic tensors. J. Mech. Phys. Solids 99, 93-115 (2017)

2. Agiasofitou, E., Lazar, M.: Conservation and balance laws in linear elasticity of grade three. J. Elast. 94, 69-85 (2009)

3. Agiasofitou, E., Lazar, M.: Anisotropic elasticity of grade three: conservation and balance laws. In: Steinmann, P. (ed.) IUTAM Symposium on Progress in the Theory and Numerics of Configurational Mechanics. IUTAM Bookseries, vol. 17, pp. 179-191. Springer, Berlin (2009)

4. Auffray, N., Le Quang, H., He, Q.C.: Matrix representations for 3D strain-gradient elasticity. J. Mech. Phys. Solids 61, 1202-1223 (2013)

5. Auffray, N., He, Q.C., Le Quang, H.: Complete symmetry classification and compact matrix representations for 3D strain gradient elasticity. Int. J. Solids Struct. 159, 197-210 (2019)

6. Barsch, G.R.: Relation between third-order elastic constants of single crystals and polycrystals. J. Appl. Phys. 39, 3780-3793 (1968)

7. Bross, H.: Berechnung der elastischen Konstanten dritter Ordnung der Alkalihalogenidkristalle. Z. Phys. 175, 345-369 (1963)

8. Castañeda, P.P., Suquet, P.: Nonlinear composites. Adv. Appl. Mech. 34(C), 171-302 (1997)

9. Cowin, S.C., Mehrabadi, M.M.: The structure of the linear anisotropic elastic symmetries. J. Mech. Phys. Solids 40, 1459$1471(1992)$

10. Csáki, F.: A concise proof of Sylvester's theorem. Period. Polytech. Electr. Eng. 14, 105-112 (1970)

11. Dederichs, P.H., Leibfried, G.: Elastic Green's function for anisotropic cubic crystals. Phys. Rev. 188, 1175-1183 (1969)

12. Dell'Isola, F., Sciarra, G., Vidoli, S.: Generalized Hooke's law for isotropic second gradient materials. Proc. R. Soc. A. 465, 2177-2196 (2009)

13. DiVincenzo, D.P.: Dispersive corrections to continuum elastic theory in cubic crystals. Phys. Rev. B 34, 5450-5465 (1986)

14. Eringen, A.C.: Microcontinuum Field Theories I: Foundations and Solids. Springer, New York (1999)

15. Gilbert, G.T.: Positive definite matrices and Sylvester's criterion. Am. Math. Mon. 98, 44-46 (1991)

16. Haussühl, S.: Die Abweichungen von den Cauchy-Relationen. Phys. kondens. Materie 6, 181-192 (1967)

17. Hermann, C.: Tensoren und Kristallsymmetrie. Z. Kristallogr. 89, 32-48 (1934)

18. Hirth, J.P., Lothe, J.: Theory of Dislocations, 2nd edn. Wiley, New York (1982)

19. Jakata, K., Every, A.G.: Determination of the dispersive elastic constants of the cubic crystals Ge, Si, GaAs, and InSb. Phys. Rev. B 77, 174301 (2008)

20. Jeffreys, H., Jeffreys, B.: Methods of Mathematical Physics. Cambridge University Press, Cambridge (1972)

21. Kalpakides, V.K., Agiasofitou, E.: On material equations in second gradient electroelasticity. J. Elast. 67, 205-227 (2002)

22. Kirchhoff, G.: Über das Gleichgewicht und die Bewegung eines unendlich dünnen elastischen Stabes. J. Reine Angew. Math. 1, 285-313 (1859)

23. Kittel, C.: Introduction to Solid State Physics. Wiley, New York (2004)

24. Kohlhaas, R., Dunner, P., Schmitz-Pranghe, N.: Über die Temperaturabhängigkeit der Gitterparameter von Eisen, Kobalt und Nickel im Bereich hoher Temperaturen. Z. Angew. Phys. 23, 245-249 (1967)

25. Kröner, E.: Kontinuumstheorie der Versetzungen und Eigenspannungen. Springer, Berlin (1958)

26. Kröner, E.: Berechnung der elastischen Konstanten des Vielkristalls aus den Konstanten des Einkristalls. Z. Phys. 151, 504-518 (1958) 
27. Lazar, M., Maugin, G.A., Aifantis, E.C.: Dislocations in second strain gradient elasticity. Int. J. Solids Struct. 43, 1787-1817 (2006)

28. Lazar, M., Maugin, G.A., Aifantis, E.C.: Addendum to: "Dislocations in second strain gradient elasticity" [Int. J. Solids Struct. 43 (2006) 1787-1817]. Int. J. Solids Struct. 47, 738-739 (2010)

29. Lazar, M., Kirchner, H.O.K.: The Eshelby stress tensor, angular momentum tensor and dilatation flux in gradient elasticity. Int. J. Solids Struct. 44, 2477-2486 (2007)

30. Lazar, M., Anastassiadis, C.: Lie point symmetries, conservation and balance laws in linear gradient elastodynamics. J. Elast. 88, 5-25 (2007)

31. Lazar, M.: Irreducible decomposition of strain gradient tensor in isotropic strain gradient elasticity. Z. Angew. Math. Mech. 96, 1291-1305 (2016)

32. Lazar, M., Po, G.: On Mindlin's isotropic strain gradient elasticity: Green tensors, regularization, and operator-split. J. Micromech. Mol. Phys. 3(3 \& 4), 1840008 (2018)

33. Lazar, M., Agiasofitou, E., Po, G.: Three-dimensional nonlocal anisotropic elasticity: a generalized continuum theory of Ångström-mechanics. Acta Mech. 231, 743-781 (2020)

34. Lee, B.-J., Baskes, M., Kim, H., Koo Cho, Y.: Second nearest-neighbor modified embedded atom method potentials for bcc transition metals. Phys. Rev. B 64, 184102 (2001)

35. Lee, B.-J.: Second nearest-neighbor modified embedded-atom-method (2NN MEAM), (2014). https://openkim.org/cite/ MD_111291751625_001

36. Leibfried, G.: Versetzugen in anisotropem material. Z. Phys. 135, 23-43 (1953)

37. Leibfried, G.: Gittertheorie der mechanischen und thermischen Eigenschaften der Kristalle. In: Kristallphysik, I., Flügge, S. (eds.) Handbuch der Physik, vol. V.I.I., pp. 104-324. Springer, Berlin (1955)

38. Ludwig, W.: Festkörperphysik. Akademische Verlagsgesellschaft, Frankfurt (1970)

39. Maranganti, R., Sharma, P.: A novel atomistic approach to determine strain-gradient elasticity constants: tabulation and comparison for various metals, semiconductors, silica, polymers and the (Ir) relevance for nanotechnologies. J. Mech. Phys. Solids 55, 1823-1852 (2007)

40. Mehrabadi, M.M., Cowin, S.C.: Eigentensors of linear anisotropic elastic materials. Q. J. Mech. Appl. Math. 43, 15-41 (1990)

41. Mindlin, R.D.: Micro-structure in linear elasticity. Arch. Ration. Mech. Anal. 16, 51-78 (1964)

42. Mindlin, R.D.: Theories of elastic continua and crystal lattice theories. In: Kröner, E. (ed.) Mechanics of Generalized Continua. IUTAM Symposium, pp. 312-320. Springer, Berlin (1968)

43. Mindlin, R.D.: Elasticity, piezoelectricity and crystal lattice dynamics. J. Elast. 2, 217-282 (1972)

44. Nye, J.F.: Physical Properties of Crystals. Oxford University Press, Oxford (1957)

45. Ojaghnezhad, F., Shodja, H.M.: A combined first principles and analytical determination of the modulus of cohesion, surface energy, and the additional constants in the second strain gradient elasticity. Int. J. Solids Struct. 50, 3967-3974 (2013)

46. Po, G., Admal, N.C., Lazar, M.: The Green tensor of Mindlin's anisotropic first strain gradient elasticity. Mater. Theory 3, 3 (2019)

47. Portigal, D.L., Burstein, E.: Acoustical activity and other first-order spatial dispersion effects in crystals. Phys. Rev. 170, 673-170 (1968)

48. Schouten, J.A.: Tensor Analysis for Physicists. Oxford University Press, Oxford (1951)

49. Shibutani, Y.: Nonlocal elastic constants of centrosymmetric homogeneous lattice structure and inhomogeneous one. Int. J. Ser. A Solid Mech. Mater. Eng. 41(4), 547-553 (1998)

50. Shodja, H.M., Moosavian, H., Ojaghnezhad, F.: Toupin-Mindlin first strain gradient theory revisited for cubic crystals of hexoctahedral class: analytical expression of the material parameters in terms of the atomic force constants and evaluation via ab initio DFT. Mech. Mater. 123, 19-29 (2018)

51. Straumanis, M.E., Yu, L.S.: Lattice parameters, densities, expansion coefficients and perfection of structure of $\mathrm{Cu}$ and of $\mathrm{Cu}-\mathrm{In} \alpha$ phase. Acta Cryst. A 25, 676-682 (1969)

52. Sunyk, R., Steinmann, P.: On higher gradients in continuum-atomistic modelling. Int. J. Solids Struct. 40, 6877-6896 (2003)

53. Thomas, T.Y.: On the stress-strain relations for cubic crystals. Proc. Natl. Acad. Sci. 55, 235-239 (1966)

54. Ting, T.C.T.: Anisotropic Elasticity: Theory and Applications. Oxford University Press, Oxford (1996)

55. Toupin, R.A.: Elastic materials with couple-stresses. Arch. Ration. Mech. Anal. 11, 385-414 (1962)

56. Toupin, R.A.: Theories of elasticity with couple-stress. Arch. Ration. Mech. Anal. 17, 85-112 (1964)

57. Waseda, Y., Hirata, K., Ohtani, M.: High-temperature thermal expansion of platinum, tantalum, molybdenum, and tungsten measured by X-ray diffraction. High Temp. High Press. 7, 221-226 (1975)

58. Witt, W.: Absolute Präzisionsbestimmung von Gitterkonstanten an Germanium- und Aluminium-Einkristallen mit Elektroneninterferenzen. Z. Naturforsch. 22A, 92-95 (1967)

Publisher's Note Springer Nature remains neutral with regard to jurisdictional claims in published maps and institutional affiliations. 